\title{
ASTEROSEISMIC FUNDAMENTAL PROPERTIES OF SOLAR-TYPE STARS OBSERVED BY THE NASA KEPLER MISSION
}

\author{
W. J. Chaplin ${ }^{1,2}$, S. Basu ${ }^{3}$, D. Huber ${ }^{4,26}$, A. Serenellit ${ }^{5}$, L. Casagrande ${ }^{6}$, V. Silva Aguirre ${ }^{2}$, W. H. Ball ${ }^{7,8}$,

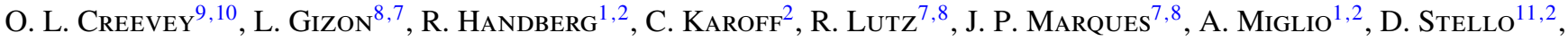 \\ M. D. Suran ${ }^{12}$, D. Pricopi ${ }^{12}$, T. S. Metcalfe ${ }^{13,2}$, M. J. P. F. G. Monteiro ${ }^{14}$, J. Molenda-ŻaKowicz ${ }^{15}$, T. ApPourChauX $^{10}$, \\ J. Christensen-DalsgaArd ${ }^{2}$, Y. Elsworth ${ }^{1,2}$, R. A. García ${ }^{16}$, G. Houdek ${ }^{2}$, H. KJeldsen ${ }^{2}$, A. Bonanno ${ }^{17}$, T. L. CAMPante ${ }^{1,2}$, \\ E. Corsaro ${ }^{18,17}$, P. Gaulme ${ }^{19}$, S. HeKKer ${ }^{8,20}$, S. Mathur ${ }^{13,21}$, B. Mosser ${ }^{22}$, C. Régulo ${ }^{23,24}$, AND D. SAlabert ${ }^{25}$ \\ ${ }^{1}$ School of Physics and Astronomy, University of Birmingham, Edgbaston, Birmingham, B15 2TT, UK \\ ${ }^{2}$ Stellar Astrophysics Centre (SAC), Department of Physics and Astronomy, Aarhus University, Ny Munkegade 120, DK-8000 Aarhus C, Denmark \\ ${ }^{3}$ Department of Physics and Astronomy, Yale University, P.O. Box 208101, New Haven, CT 06520, USA \\ ${ }^{4}$ NASA Ames Research Center, MS 244-30, Moffett Field, CA 94035, USA \\ ${ }^{5}$ Instituto de Cièncias del Espacio (CSIC-IEEC), Facultad de Ciències, Campus UAB, E-08193 Bellaterra, Spain \\ ${ }^{6}$ Research School of Astronomy and Astrophysics, Mount Stromlo Observatory, The Australian National University, ACT 2611, Australia \\ ${ }^{7}$ Institut für Astrophysik, Georg-August-Universität Göttingen, D-37077 Göttingen, Germany \\ ${ }^{8}$ Max-Planck-Institut für Sonnensystemforschung, D-37191 Katlenburg-Lindau, Germany \\ ${ }^{9}$ Université de Nice Sophia-Antipolis, Laboratoire Lagrange, UMR 7293, CNRS, Observatoire de la Côte d'Azur, F-06304 Nice, France \\ ${ }^{10}$ Institut d'Astrophysique Spatiale, Université Paris XI-CNRS (UMR8617), Batiment 121, F-91405 Orsay Cedex, France \\ ${ }^{11}$ Sydney Institute for Astronomy, School of Physics, University of Sydney, Sydney, Australia \\ ${ }^{12}$ Astronomical Institute of the Romanian Academy, Str. Cutitul de Argint, 5, RO 40557 Bucharest, Romania \\ ${ }^{13}$ Space Science Institute, 4750 Walnut Street, Suite 205, Boulder, CO 80301, USA \\ ${ }^{14}$ Centro de Astrofísica, Universidade do Porto, Rua das Estrelas, 4150-762 Porto, Portugal \\ 15 Astronomical Institute, University of Wrocław, ul. Kopernika, 11, 51-622 Wrocław, Poland \\ ${ }^{16}$ Laboratoire AIM, CEA/DSM, CNRS, Université Paris Diderot, IRFU/SAp, F-91191 Gif-sur-Yvette Cedex, France \\ ${ }^{17}$ INAF-Astrophysical Observatory of Catania, Via S. Sofia 78, I-95123 Catania, Italy \\ ${ }^{18}$ Instituut voor Sterrenkunde, KU Leuven, Celestijnenlaan 200D, B-3001 Leuven, Belgium \\ ${ }^{19}$ Department of Astronomy, New Mexico State University, P.O. Box 30001, MSC 4500, Las Cruces, NM 88003-8001, USA \\ ${ }^{20}$ Astronomical Institute "Anton Pannekoek," University of Amsterdam, Science Park 904, 1098 XH Amsterdam, The Netherlands \\ ${ }^{21}$ High Altitude Observatory, NCAR, P.O. Box 3000, Boulder, CO 80307, USA \\ ${ }^{22}$ LESIA, CNRS, Université Pierre et Marie Curie, Université Denis Diderot, Observatoire de Paris, F-92195 Meudon Cedex, France \\ ${ }^{23}$ Instituto de Astrofísica de Canarias, E-38200 La Laguna, Tenerife, Spain \\ ${ }^{24}$ Departamento de Astrofísica, Universidad de La Laguna, E-38206 La Laguna, Tenerife, Spain \\ ${ }^{25}$ Laboratoire Lagrange, UMR7293, Université de Nice Sophia-Antipolis, CNRS, Observatoire de la Côte d'Azur, Bd. de Observatoire, F-06304 Nice, France \\ Received 2013 May 1; accepted 2013 October 3; published 2013 December 11
}

\begin{abstract}
We use asteroseismic data obtained by the NASA Kepler mission to estimate the fundamental properties of more than 500 main-sequence and sub-giant stars. Data obtained during the first 10 months of Kepler science operations were used for this work, when these solar-type targets were observed for one month each in survey mode. Stellar properties have been estimated using two global asteroseismic parameters and complementary photometric and spectroscopic data. Homogeneous sets of effective temperatures, $T_{\text {eff }}$, were available for the entire ensemble from complementary photometry; spectroscopic estimates of $T_{\text {eff }}$ and $[\mathrm{Fe} / \mathrm{H}]$ were available from a homogeneous analysis of ground-based data on a subset of 87 stars. We adopt a grid-based analysis, coupling six pipeline codes to 11 stellar evolutionary grids. Through use of these different grid-pipeline combinations we allow implicitly for the impact on the results of stellar model dependencies from commonly used grids, and differences in adopted pipeline methodologies. By using just two global parameters as the seismic inputs we are able to perform a homogenous analysis of all solar-type stars in the asteroseismic cohort, including many targets for which it would not be possible to provide robust estimates of individual oscillation frequencies (due to a combination of low signal-to-noise ratio and short dataset lengths). The median final quoted uncertainties from consolidation of the grid-based analyses are for the full ensemble (spectroscopic subset) approximately $10.8 \%$ (5.4\%) in mass, $4.4 \%$ (2.2\%) in radius, 0.017 dex ( $0.010 \mathrm{dex})$ in $\log g$, and $4.3 \%(2.8 \%)$ in mean density. Around 36\% (57\%) of the stars have final age uncertainties smaller than 1 Gyr. These ages will be useful for ensemble studies, but should be treated carefully on a star-bystar basis. Future analyses using individual oscillation frequencies will offer significant improvements on up to 150 stars, in particular for estimates of the ages, where having the individual frequency data is most important.
\end{abstract}

Key words: asteroseismology - methods: data analysis - stars: fundamental parameters - stars: interiors

Online-only material: color figures, machine-readable tables

\section{INTRODUCTION}

Recent advances in observational asteroseismology are making it possible to estimate accurate and precise fundamental properties of a growing number of solar-type stars. These advances have come in large part from new satellite observations,

${ }^{26}$ NASA Postdoctoral Program Fellow. for example from the French-led CoRoT satellite (e.g., Michel et al. 2008; Appourchaux et al. 2008; Michel \& Baglin 2012), and in particular the NASA Kepler mission (Gilliland et al. 2010a).

During the first 10 months of science operations more than 2000 solar-type stars were selected by the Kepler Asteroseismic 
Science Consortium (KASC) to be observed as part of an asteroseismic survey of the Sun-like population in the Kepler field of view. Solar-like oscillations were detected by Kepler in more than 500 stars (Chaplin et al. 2011), and from these data robust global or average asteroseismic parameters were determined for all targets in the sample. These asteroseismic parameters allow us to estimate fundamental properties of the stars. In this paper we present stellar properties-namely masses, radii, surface gravities, mean densities and ages-of this asteroseismic sample of main-sequence and subgiant stars.

The most precise asteroseismically derived stellar properties are obtained when the frequencies of individual modes of oscillation are modeled (see, e.g., Metcalfe et al. 2010, 2012; Silva Aguirre et al. 2013). Recent noteworthy examples have included several solar-type exoplanet host stars observed by Kepler (e.g., see Batalha et al. 2011; Howell et al. 2012; Carter et al. 2012; Gilliland et al. 2013; Chaplin et al. 2013). When the signal-to-noise ratios $(\mathrm{S} / \mathrm{Ns})$ in the asteroseismic data are too low to allow robust fitting of individual mode frequencies, or the frequency resolution is insufficient to resolve clearly the mode structure, it is nevertheless still possible to extract average or global asteroseismic parameters. The main parameters are the average large frequency separation, $\Delta v$, and the frequency of maximum oscillations power, $v_{\max }$. Automated analysis codes developed for application to Kepler data (e.g., see Chaplin et al. 2008; Stello et al. 2009) have enabled efficient extraction of these parameters on large numbers of stars, even at quite low $\mathrm{S} / \mathrm{N}$ levels.

Here, the use of global asteroseismic parameters has allowed us to determine the properties of over 500 stars, rather than the properties of just the smaller cohort of around 150 stars observed continuously over long periods by Kepler for which robust frequencies may be determined. Detailed studies of several Kepler targets have shown that results obtained using only global parameters provide a good match (within their uncertainties) to those given by analysis of individual frequencies (e.g., see exoplanet host-star references above; also Mathur et al. 2012; Metcalfe et al. 2012; Doğan et al. 2013; Silva Aguirre et al. 2013), although fractional uncertainties in the estimated properties are usually inferior (most notably the uncertainties in the estimated ages).

We use a grid-based method to determine stellar properties, but with the powerful diagnostic information contained in the seismic parameters also brought to bear. This is the classic approach of matching the observations to well-sampled grids of stellar evolutionary models (tracks, or isochrones). It is not uncommon in the stellar literature for grid-based estimates of stellar properties to be presented from analyses that involve one pipeline code coupled to only one grid of models (with a given input physics). We adopt the grid-based analysis, but here we couple six pipeline codes to 11 stellar evolutionary grids. By using a range of grid-pipeline combinations-comprising a selection of widely used stellar evolutionary models, covering a range of commonly adopted input physics-we allow implicitly in our final estimates for the impact of stellar model dependencies from commonly used grids and physics, and also differences in adopted analysis pipeline methodologies.

\section{DETERMINATION OF STELLAR PROPERTIES USING GLOBAL ASTEROSEISMIC PARAMETERS}

\subsection{Global Asteroseismic Parameters}

Cool subgiants and low-mass, main-sequence stars show rich spectra of solar-like oscillations, small-amplitude pulsations that
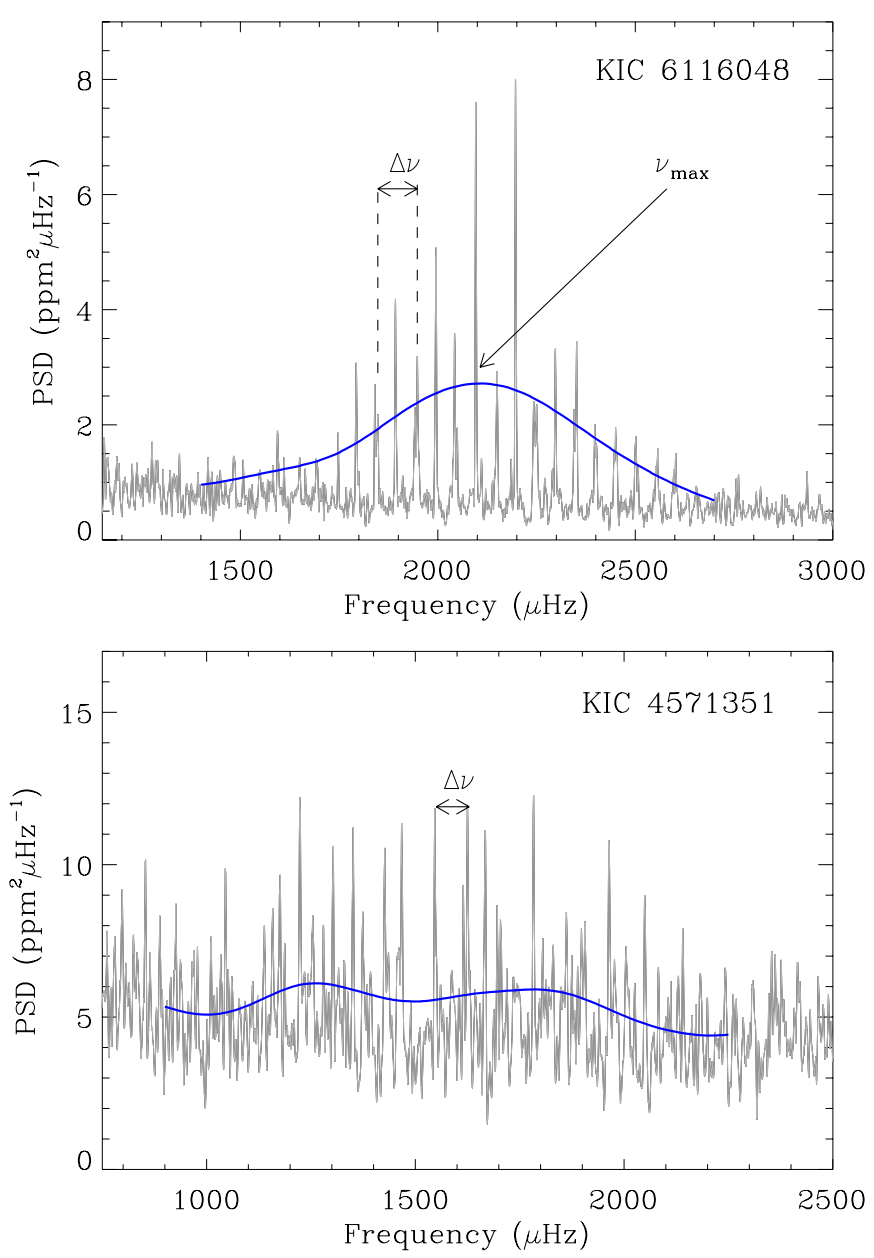

Figure 1. Top panel: frequency power spectrum of KIC 6116048, computed from one month of Kepler data. The blue line shows the envelope of power given by the oscillations, from heavily smoothing the power spectrum (and here multiplied by a factor of five to show the envelope more clearly). Bottom panel: frequency power spectrum of the much fainter KIC 4571351. The higher levels of shot noise compared to KIC 6116048 lead to much lower levels of S/N in the oscillations spectrum, making it difficult to extract a robust estimate of $v_{\max }$.

(A color version of this figure is available in the online journal.)

are excited and damped intrinsically by convection in the outer parts of the star. The most prominent oscillations are acoustic (pressure, or $p$ ) modes of high radial order, $n$. The observed power in the oscillations is modulated in frequency by an envelope that typically has an approximately Gaussian shape (as can be seen in Figure 1). The frequency of maximum oscillations power, $v_{\max }$, has been shown to scale to good approximation as $g T_{\text {eff }}^{-1 / 2}$ (Brown et al. 1991; Kjeldsen \& Bedding 1995; Chaplin et al. 2008; Belkacem et al. 2011), where $g$ is the surface gravity and $T_{\text {eff }}$ is the effective temperature of the star. The radial order at $v_{\max }$ ranges from $n \simeq 15$ to 19 in sub-giants, and $n \simeq 17$ to 25 in main-sequence stars. The transition from the main-sequence to the sub-giant phase occurs at approximately $v_{\max }=2000 \mu \mathrm{Hz}$ in stars of solar mass and composition; this frequency decreases to approximately $800 \mu \mathrm{Hz}$ at $M \simeq 1.5 M_{\odot}$.

The most obvious frequency spacings in the spectrum are the large frequency separations, $\Delta v$, between consecutive overtones $n$ of the same spherical angular degree, $l$. The average large separation scales to very good approximation as $\rho^{1 / 2}, \rho \propto$ $M / R^{3}$ being the mean density of a star having mass $M$ and surface radius $R$ (e.g., see Tassoul 1980; Ulrich 1986; Christensen-Dalsgaard 1993). 


\subsection{Principles of Stellar Property Estimation}

As mentioned earlier, we use grid-based methods to determine the stellar properties, but unlike earlier works we use seismic observables - here, the global parameters $\Delta v$ and $v_{\max }$ - together with non-seismic inputs-here, complementary estimates of $T_{\text {eff }}$ and the metallicity $[\mathrm{Fe} / \mathrm{H}]$ — to determine stellar properties.

The information encoded in $\Delta v$ may be employed in one of two ways. One may compute theoretical oscillation frequencies of each model in the grid, and from those frequencies calculate a suitable average $\Delta v$ for comparison with the observations, e.g., from $l=0$ (radial-mode) frequencies spanning the same orders $n$ as those detected in the data. Alternatively, one may circumvent the need to compute individual oscillation frequencies of every model and instead use the dependence of $\Delta v$ on the mean stellar density (Section 2.1) as a scaling relation normalized by solar properties and parameters, i.e.,

$$
\frac{\Delta v}{\Delta v_{\odot}} \simeq \sqrt{\frac{M / M_{\odot}}{\left(R / R_{\odot}\right)^{3}}}
$$

The fundamental properties of the models (i.e., $R$ and $M$ ) are thence used as inputs to Equation (1) to calculate modelvalues of $\Delta v$, against which differences with the observations may be computed. Comparison of predictions of Equation (1) with predictions of $\Delta v$ from model-calculated eigenfrequencies reveal small but systematic offsets for solar-type stars, which can be as large as $\simeq 2 \%$ (e.g., see Ulrich 1986; White et al. 2011). When plotted as a function of $T_{\text {eff }}$, these differences manifest as a "boomerang" shaped trend (cf. Figures 5 and 6 of White et al. 2011). The median uncertainty in $\Delta v$ in our sample is $2.1 \%$. As we shall see later, this effect is clearly detectable in our results.

One phenomenon that none of the above allows for is the impact of poor modeling of the near-surface layers of stars. In the case of the Sun, this has all been shown to lead to a frequency dependent offset between observed $p$-mode frequencies and the model-predicted $p$-mode frequencies (e.g., see Christensen-Dalsgaard \& Gough 1984; Dziembowski et al. 1988; Christensen-Dalsgaard \& Thompson 1997; Kjeldsen et al. 2008; Chaplin \& Miglio 2013, and references therein), with the model frequencies being on average too high by a few $\mu \mathrm{Hz}$. This offset, which is sometimes called the "surface term," is larger in modes at higher frequencies. The average large frequency separation will also be affected by the surface term, by an amount that depends on the gradient of the frequency offset with radial order, $n$. In the case of the Sun, the model-predicted $\Delta v$ is about $0.75 \%$ higher than the observed $\Delta v$. The impact on grid-search results for the Sun is relatively small, about $0.3 \%$ in the inferred radius, and not a cause for concern given the level of the uncertainties in the global asteroseismic parameters used here (e.g., see Basu et al. 2010). Offsets for other solar-type stars would have to be substantially larger than the solar offsets to give significant bias in our results. However, we still await definitive results on the surface-term offsets, although existing studies suggest that offsets may be Sun-like in size when stars have close-to-solar surface properties (e.g., Kjeldsen et al. 2008; Mathur et al. 2012; Gruberbauer et al. 2013).

Information encoded in $v_{\max }$ is currently employed only in scaling-relation form, i.e., with reference to Section 2.1 we have

$$
\frac{v_{\max }}{v_{\max , \odot}} \simeq \frac{M / M_{\odot}}{\left(R / R_{\odot}\right)^{2} \sqrt{\left(T_{\mathrm{eff}} / T_{\mathrm{eff}, \odot}\right)}}
$$

so that again it is the properties of the models $\left(R, M, T_{\text {eff }}\right)$ that yield model-values of $v_{\max }$, against which the observations are compared.

The form of the above equations of course indicates that we do not necessarily need to employ a grid of stellar evolutionary models: if $\Delta v, v_{\max }$ and $T_{\text {eff }}$ are known for a star, Equations (1) and (2) may be used directly to infer the stellar radius, mass, density and surface gravity (though not the age). This socalled direct method gives uncertainties that are larger than those given by the grid-based approach because the scaling relations are not constrained by the equations governing stellar structure and evolution, i.e., at a given mass and radius any value of $T_{\text {eff }}$ is possible. However, we know from stellar evolution theory that only a narrow range of $T_{\text {eff }}$ is allowed (and moreover, the effective temperature also depends on the chemical composition). Employing a grid of stellar models hence gives smaller uncertainties.

A good deal of effort has recently been devoted to testing the accuracy of the scaling relations and asteroseismically inferred properties, using results on stars where it is possible to independently estimate the properties to a verifiable (high) level of accuracy (e.g., stars in binaries). For a comprehensive review, see Chaplin \& Miglio (2013) and references therein. Examples include comparisons with properties estimated using eclipsing binaries, stellar parallaxes, long baseline interferometry, and members of open clusters (e.g., Stello et al. 2008; Bedding 2011; Brogaard et al. 2012; Miglio 2012; Miglio et al. 2012; Huber et al. 2012; Silva Aguirre et al. 2012). Good agreement has been found for main-sequence stars and sub-giants, with no evidence for systematic deviations found at the level of the observational uncertainties, i.e., upper limits of around $4 \%$ in radius and $10 \%$ in mass. But further tests are needed, in particular tests of the estimated masses.

Mosser et al. (2013) have also recently advocated modifying the observed average $\Delta v$, for use with the scaling relations, to the value expected in the high-frequency asymptotic limit. We test the impact of their suggested modifications in Section 5.

Most grid-based methods determine the characteristics of stars by finding the maximum of the likelihood function of a set of input parameters, $\left\{v_{\max }, \Delta v, T_{\text {eff }},[\mathrm{Fe} / \mathrm{H}]\right\}$, calculated with respect to a grid of stellar evolutionary models. The details of how this is achieved varies, depending on the pipeline used. In this work we use six different pipelines that search within 11 stellar evolutionary grids. Some pipelines used modelcalculated eigenfrequencies to estimate the model $\Delta v$, while a majority used the $\Delta v$ scaling relation. All pipelines used the $v_{\max }$ scaling relation. The listed stellar properties that we present later come from one of the pipelines that used model-calculated eigenfrequencies. The pipelines used are described further in Section 4. Characteristics and systematic biases involved with grid-based analyses have been investigated in detail by Gai et al. (2011), Basu et al. (2012), Bazot et al. (2012), and Gruberbauer et al. (2012). Systematics related to estimation of $\log g$ have recently been explored by Creevey et al. (2013).

\section{INPUT SEISMIC AND NON-SEISMIC DATA}

We used asteroseismic data on solar-type stars observed by Kepler during the first 10 months of science operations. About 2000 stars, down to Kepler apparent magnitude $K_{p} \simeq$ 12.5, were selected as potential solar-type targets based upon complementary data from the Kepler Input Catalog (KIC; Brown et al. 2011). Each target was observed continuously for one month at a short cadence of $58.85 \mathrm{~s}$ (Gilliland et al. 


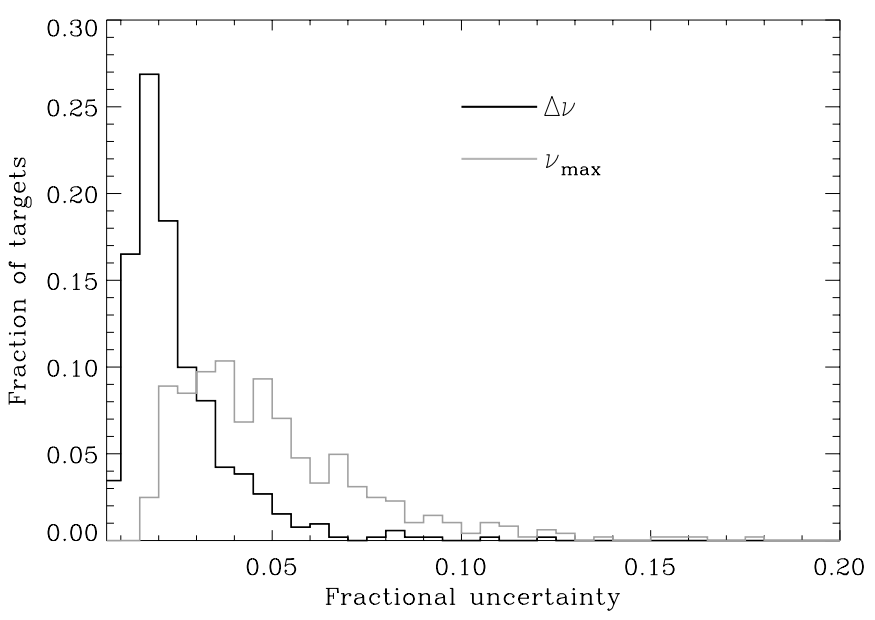

Figure 2. Histograms of fractional uncertainties for the global asteroseismic input parameters $\Delta v$ and $v_{\max }$ (see figure legend).

2010b; Jenkins et al. 2010). Timeseries were prepared for asteroseismic analysis in the manner described by García et al. (2011). Different teams attempted to detect, and then extract the basic properties of, the solar-like oscillations using automated analysis pipelines developed and extensively tested (e.g., see Bonanno et al. 2008; Huber et al. 2009; Mosser \& Appourchaux 2009; Roxburgh 2009; Campante et al. 2010; Hekker et al. 2010; Mathur et al. 2010) for application to the large ensemble of targets observed by Kepler. The basic results of this survey were presented in Chaplin et al. (2011) and Verner et al. (2011).

Figure 1 shows examples of the oscillation spectra of two stars from the survey. The top panel is a high-S/N case, where the individual modes are easily distinguishable in the spectrum. The blue line shows the envelope of power given by the oscillations (from heavily smoothing the power spectrum, and here multiplied by a factor of five to show the envelope more clearly). In this case it is trivial to extract the average large separation, $\Delta v$, and the frequency of maximum oscillations power, $v_{\max }$. The bottom panel presents a much harder, low-S/N case. Here, the $\mathrm{S} / \mathrm{N}$ is much reduced because the star is over two magnitudes fainter than the target in the top panel. Whilst it is still possible to extract a robust estimate of $\Delta v$, beating of the oscillation signal with the background means that: first, it would be much harder to extract precise estimates of the individual mode frequencies; but also, second, and of direct relevance to this paper, it is no longer possible to extract a welldefined estimate of $v_{\max }$. This was also the case for another 36 stars in the full cohort. Their properties, and the properties of the star in the bottom panel of Figure 1, were therefore derived with only $\Delta v$ used as seismic input.

We used estimates of $\Delta v$ and $v_{\max }$ returned by five teams. Full details of the pipelines may be found in Verner et al. (2011), including a discussion of the excellent level of agreement found between the results delivered by the different codes. The final parameters selected for use by the grid-search pipelines were those returned by the code described in Huber et al. (2009). Its results had the smallest average deviation from the median values in a global comparison made over all the stars. Final median fractional uncertainties were $2.1 \%$ in $\Delta v$ and $4.6 \%$ in $v_{\max }$. The distribution of fractional uncertainties in the two seismic quantities can be seen in Figure 2.

A homogeneous set of effective temperatures, $T_{\text {eff }}$, was estimated for the entire ensemble using available complementary photometry. One set of temperatures was derived by using an
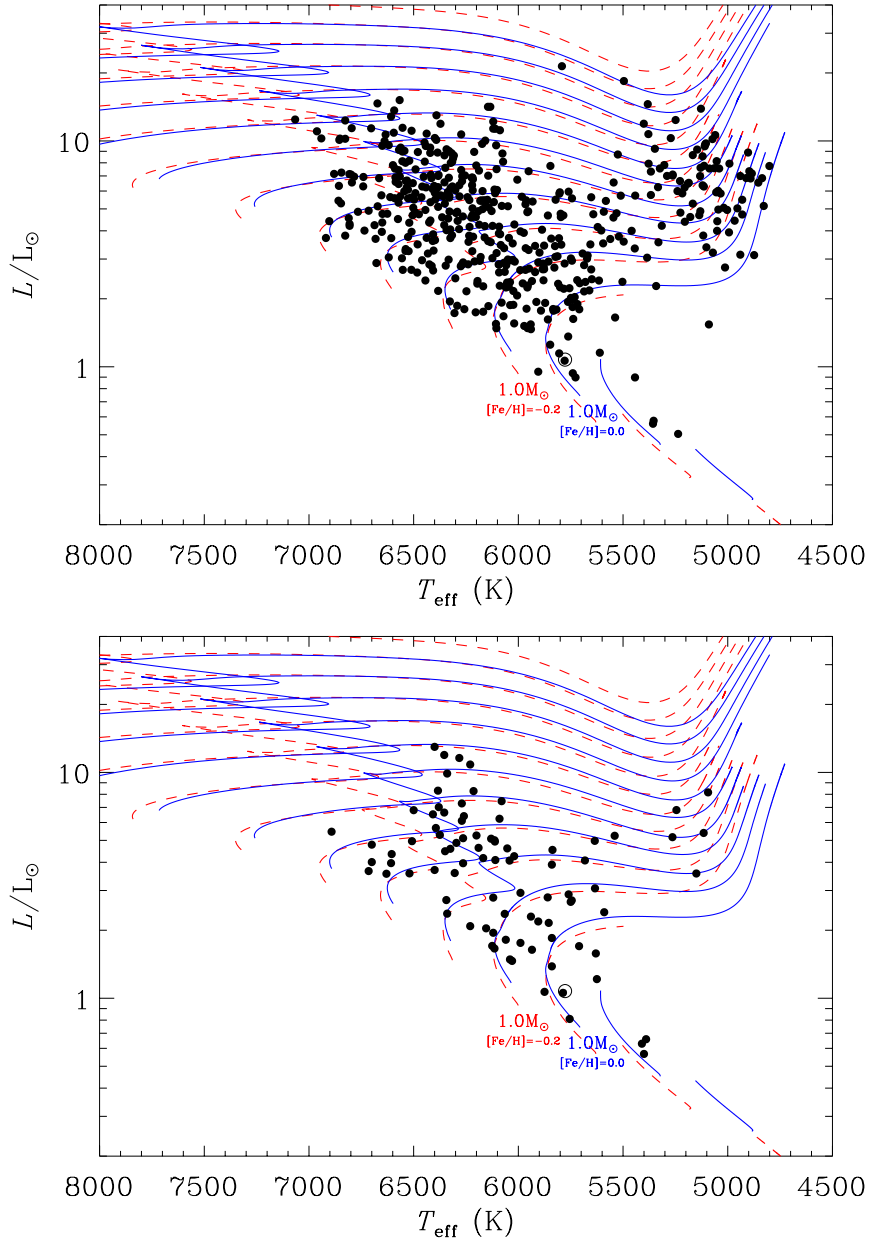

Figure 3. Top panel: HR diagram of the full cohort of stars. Temperatures are from the IRFM set; luminosities were calculated using the asteroseismically estimated stellar radii presented later in the paper, in Table 5. Bottom panel: HR diagram of the smaller cohort with spectroscopic $T_{\text {eff }}$ and $[\mathrm{Fe} / \mathrm{H}]$; luminosities were estimated using the radii presented in Table 6 . The evolutionary tracks in both panels were computed at $0.1 M_{\odot}$ intervals with the YREC code. Tracks in blue (solid lines) are for solar composition, those in red (dashed lines) for $[\mathrm{Fe} / \mathrm{H}]=-0.2$. The models are not "solar calibrated" models and hence the $1 M_{\odot}$ tracks need not pass through the exact location of the Sun.

(A color version of this figure is available in the online journal.)

Infra-Red Flux Method (IRFM) calibration (Casagrande et al. 2010; see also Silva Aguirre et al. 2012). This made use of multi-band $J H K$ photometry from the Two Micron All Sky Survey (Skrutskie et al. 2006), photometry in the Sloan Digital Sky Survey (SDSS) griz bands available in the KIC, and reddening estimates from Drimmel et al. (2003). A second set of temperatures were those derived by Pinsonneault et al. (2012), who performed a recalibration of the KIC photometry in the SDSS griz filters, using YREC models. The complementary photometry that was available to us did not allow strong constraints to be placed on the metallicity of all the targets. When using the photometric $T_{\text {eff }}$ in the grid searches we therefore adopted an $[\mathrm{Fe} / \mathrm{H}]$ corresponding to an average value for the field of $-0.2 \pm 0.3$ dex (e.g., see Silva Aguirre et al. 2011). It is worth adding that for the IRFM, the dependence of the temperatures on $[\mathrm{Fe} / \mathrm{H}]$ is rather weak. For the SDSS calibration, a change of $\delta[\mathrm{Fe} / \mathrm{H}] \simeq 0.4$ is needed to change the temperatures at approximately the $1 \sigma$ level (see Table 3 of Pinsonneault et al. 2012).

The top panel of Figure 3 shows the positions of the full cohort of stars on an HR diagram. The temperatures are from 

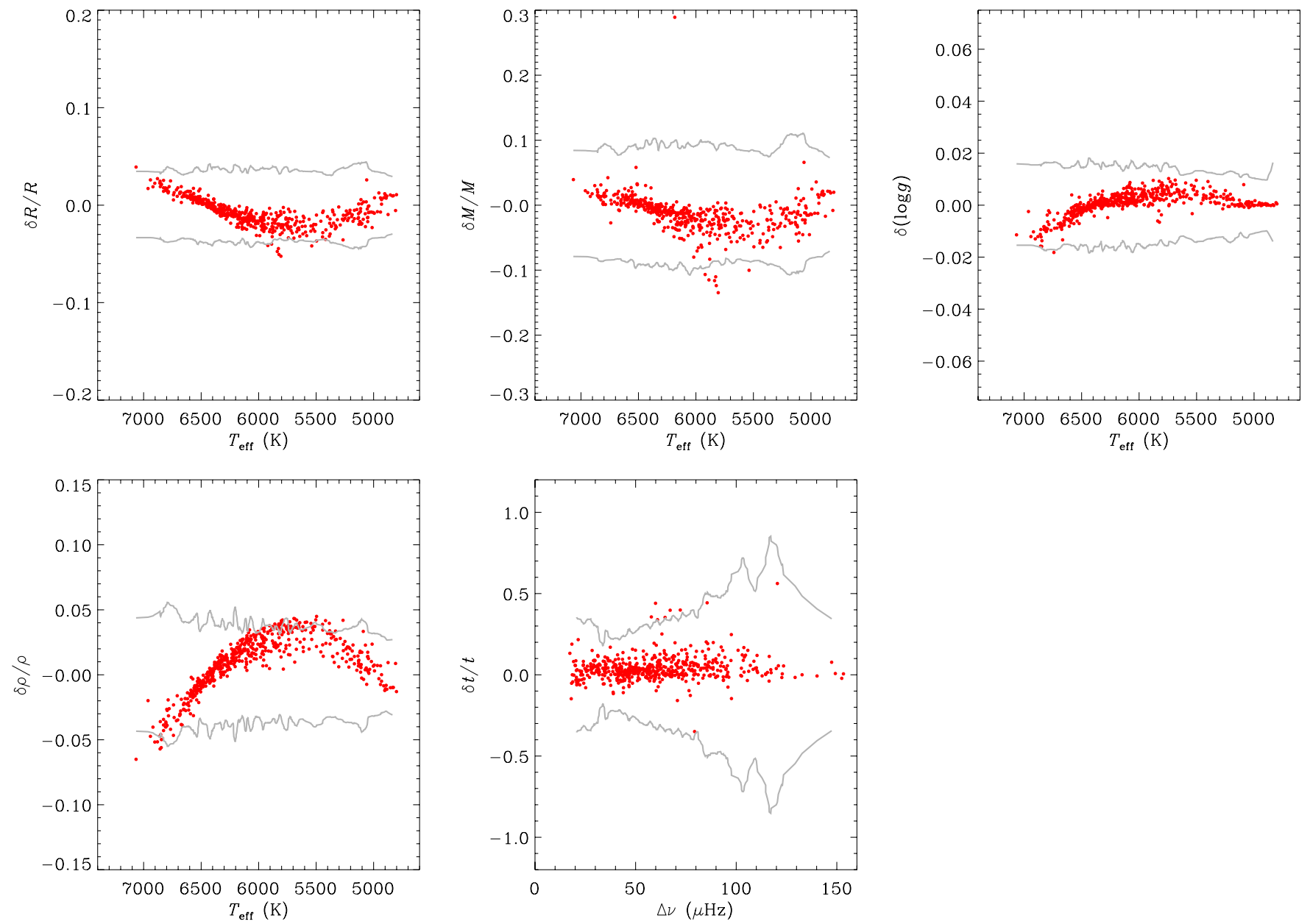

Figure 4. Fractional differences in estimated stellar properties for analyses performed by BeSPP with the GARSTEC grid, for the entire ensemble with $\Delta v$ and $v_{\max }$, the photometric (IRFM) $T_{\text {eff }}$ and field $[\mathrm{Fe} / \mathrm{H}]$ values used as inputs. The plots show differences between using model-calculated eigenfrequencies to estimate the $\Delta \nu$ of each model and using the $\Delta v$ scaling relation (in the sense scaling minus frequencies). Gray lines mark the median $1 \sigma$ envelope of the grid-pipeline returned, formal uncertainties. These lines are included to help judge the typical precision only.

(A color version of this figure is available in the online journal.)

the IRFM set, and the luminosities were calculated using the asteroseismically estimated stellar radii presented later in the paper.

Homogeneous sets of spectroscopic $T_{\text {eff }}$ and $[\mathrm{Fe} / \mathrm{H}]$ were available on a subset of 87 stars, from the data reductions performed by Bruntt et al. (2012) on high-resolution spectra obtained with the ESPaDOnS spectrograph at the $3.6 \mathrm{~m}$ Canada-France-Hawaii Telescope, and with the NARVAL spectrograph mounted on the $2 \mathrm{~m}$ Bernard Lyot Telescope at the Pic-du-Midi Observatory in France. To account for systematic differences between spectroscopic methods, we followed the procedure suggested by Torres et al. (2012) and added in quadrature $59 \mathrm{~K}$ to all $T_{\text {eff }}$ uncertainties and 0.062 dex to all $[\mathrm{Fe} / \mathrm{H}]$ uncertainties given by Bruntt et al. (2012). The distribution of this smaller sample of stars is plotted in HR form in the bottom panel of Figure 3.

All grid-modeling pipelines mentioned below were required to determine stellar properties using $\left\{\Delta v, v_{\max }, T_{\text {eff }},[\mathrm{Fe} / \mathrm{H}]\right\}$ as inputs. These seismic and non-seismic input parameters for the grid modeling are listed in Tables 1 and 2 . As noted above, for stars where $v_{\max }$ was uncertain, only $\left\{\Delta v, T_{\text {eff }},[\mathrm{Fe} / \mathrm{H}]\right\}$ were used. All pipelines were asked to use $\Delta v_{\odot}=135.1 \mu \mathrm{Hz}$ and $v_{\max , \odot}=3090 \mu \mathrm{Hz}$, which are the reference values for the Huber et al. (2009) pipeline derived from the analysis of
Table 1

Seismic and Non-seismic Input Parameters for the Full Cohort, with Photometric SDSS-calibrated and IRFM $T_{\text {eff }}$ and Field-average $[\mathrm{Fe} / \mathrm{H}]$

\begin{tabular}{lrcccc}
\hline \hline KIC & $\begin{array}{c}v_{\max } \\
(\mu \mathrm{Hz})\end{array}$ & $\begin{array}{c}\Delta v \\
(\mu \mathrm{Hz})\end{array}$ & $\begin{array}{c}\text { SDSS } T_{\text {eff }} \\
(\mathrm{K})\end{array}$ & $\begin{array}{c}\text { IRFM } T_{\text {eff }} \\
(\mathrm{K})\end{array}$ & $\begin{array}{c}{[\mathrm{Fe} / \mathrm{H}]} \\
(\mathrm{dex})\end{array}$ \\
\hline 1430163 & $1867 \pm 92$ & $84.6 \pm 2.0$ & $6796 \pm 78$ & $6806 \pm 177$ & $-0.20 \pm 0.30$ \\
1435467 & $1295 \pm 52$ & $70.8 \pm 0.8$ & $6433 \pm 86$ & $6521 \pm 164$ & $-0.20 \pm 0.30$ \\
1725815 & $1045 \pm 47$ & $55.4 \pm 1.3$ & $6550 \pm 82$ & $6532 \pm 165$ & $-0.20 \pm 0.30$ \\
2010607 & $675 \pm 86$ & $42.5 \pm 1.7$ & $6361 \pm 71$ & $6796 \pm 175$ & $-0.20 \pm 0.30$ \\
2309595 & $643 \pm 20$ & $39.3 \pm 2.2$ & $5238 \pm 65$ & $5315 \pm 112$ & $-0.20 \pm 0.30$ \\
2450729 & $1053 \pm 68$ & $61.9 \pm 0.6$ & $6029 \pm 59$ & $6093 \pm 152$ & $-0.20 \pm 0.30$ \\
2837475 & $1630 \pm 54$ & $75.1 \pm 1.3$ & $6688 \pm 57$ & $6715 \pm 174$ & $-0.20 \pm 0.30$ \\
2849125 & $728 \pm 27$ & $41.4 \pm 1.8$ & $6158 \pm 56$ & $6421 \pm 154$ & $-0.20 \pm 0.30$ \\
2852862 & $1030 \pm 38$ & $53.8 \pm 0.7$ & $6417 \pm 58$ & $6572 \pm 172$ & $-0.20 \pm 0.30$ \\
2865774 & $1252 \pm 90$ & $62.7 \pm 2.7$ & $6074 \pm 63$ & $6187 \pm 153$ & $-0.20 \pm 0.30$ \\
\hline
\end{tabular}

(This table is available in its entirety in a machine-readable form in the online journal. A portion is shown here for guidance regarding its form and content.)

VIRGO/SOHO Sun-as-a-star data (Huber et al. 2011). The uncertainties in $\Delta v_{\odot}(0.1 \mu \mathrm{Hz})$ and $v_{\max , \odot}(30 \mu \mathrm{Hz})$ were accounted for by increasing the uncertainties in $\Delta v$ and $v_{\max }$ by simple error propagation. 

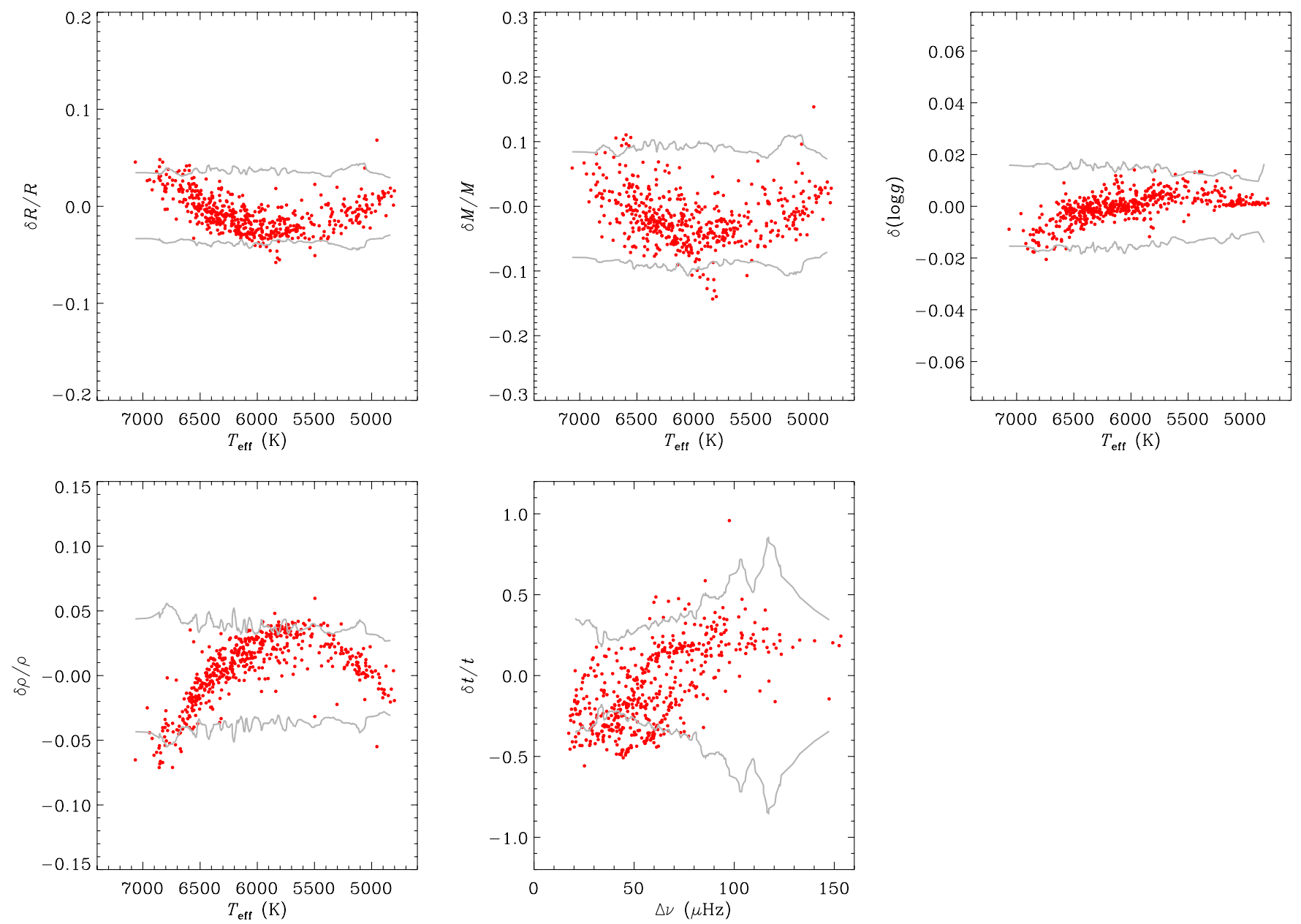

Figure 5. Fractional differences in estimated stellar properties between BeSPP/BASTI (scaling mode) and BeSPP/GARSTEC (run in frequency mode). Results shown for the entire ensemble, with $\Delta v$ and $v_{\max }$, the photometric (IRFM) $T_{\text {eff }}$ and field $[\mathrm{Fe} / \mathrm{H}]$ values used as inputs). Plot style as per Figure 4.

(A color version of this figure is available in the online journal.)

Table 2

Seismic and Non-seismic Input Parameters for the Sample with Spectroscopic $T_{\text {eff }}$ and $[\mathrm{Fe} / \mathrm{H}]$ from Bruntt et al. (2012)

\begin{tabular}{|c|c|c|c|c|}
\hline$\overline{\mathrm{KIC}}$ & $\begin{array}{c}v_{\max } \\
(\mu \mathrm{Hz})\end{array}$ & $\begin{array}{c}\Delta v \\
(\mu \mathrm{Hz})\end{array}$ & $\begin{array}{l}T_{\text {eff }} \\
(\mathrm{K})\end{array}$ & $\begin{array}{c}{[\mathrm{Fe} / \mathrm{H}]} \\
(\mathrm{dex})\end{array}$ \\
\hline 1430163 & $1867 \pm 92$ & $84.6 \pm 2.0$ & $6520 \pm 84$ & $-0.11 \pm 0.09$ \\
\hline 1435467 & $1295 \pm 52$ & $70.8 \pm 0.8$ & $6264 \pm 84$ & $-0.01 \pm 0.09$ \\
\hline 2837475 & $1630 \pm 54$ & $75.1 \pm 1.3$ & $6700 \pm 84$ & $-0.02 \pm 0.09$ \\
\hline 3424541 & $745 \pm 55$ & $41.5 \pm 1.1$ & $6080 \pm 84$ & $0.01 \pm 0.09$ \\
\hline 3427720 & $2756 \pm 191$ & $119.9 \pm 2.0$ & $6040 \pm 84$ & $-0.03 \pm 0.09$ \\
\hline 3456181 & $921 \pm 30$ & $52.0 \pm 0.8$ & $6270 \pm 84$ & $-0.19 \pm 0.09$ \\
\hline 3632418 & $1159 \pm 44$ & $60.9 \pm 0.4$ & $6190 \pm 84$ & $-0.16 \pm 0.09$ \\
\hline 3656476 & $1887 \pm 40$ & $93.3 \pm 1.3$ & $5710 \pm 84$ & $0.34 \pm 0.09$ \\
\hline 3733735 & $1974 \pm 121$ & $92.8 \pm 2.5$ & $6715 \pm 84$ & $-0.04 \pm 0.09$ \\
\hline 4586099 & $1146 \pm 50$ & $61.5 \pm 0.9$ & $6296 \pm 84$ & $-0.17 \pm 0.09$ \\
\hline
\end{tabular}

(This table is available in its entirety in a machine-readable form in the online journal. A portion is shown here for guidance regarding its form and content.)

\section{GRID-BASED PIPELINES: DETAILS}

We used six different grid-based pipelines to determine stellar properties:

1. Yale-Birmingham (YB; Basu et al. 2010, 2012; Gai et al. 2011);

2. Bellaterra Stellar Properties Pipeline (BeSPP; A. Serenelli et al. 2013, in preparation);
3. RadEx10 (Creevey et al. 2013);

4. RADIUS (Stello et al. 2009);

5. SEEK (Quirion et al. 2010); and

6. Göttingen (GOE; W. H. Ball et al., in preparation, details below).

Further details of how most of the pipelines work are available in the literature. Here, we provide brief outlines.

The YB pipeline is based on finding the maximum likelihood of the set of input parameter data calculated with respect to the grid of models. For a given observational (central) input parameter set, the first key step in the method is to generate 10,000 input parameter sets by adding different random realizations of Gaussian noise, commensurate with the observational uncertainties, to the actual (central) observational input parameter set. The distribution of any property, say radius, is then obtained from the central parameter set and the 10,000 perturbed parameter sets, which form the distribution function. The final estimate of the property is the median of this distribution. The $1 \sigma$ limits from the median are adopted as measures of the uncertainties. The BeSPP and RadEx 10 pipelines both employ the same principles as the YB pipeline. They differ in some minor details, and also in whether the mean or the median of the distribution function is used as the adopted value of the property. We have verified that this choice does not have a significant impact on the results presented in this paper. 

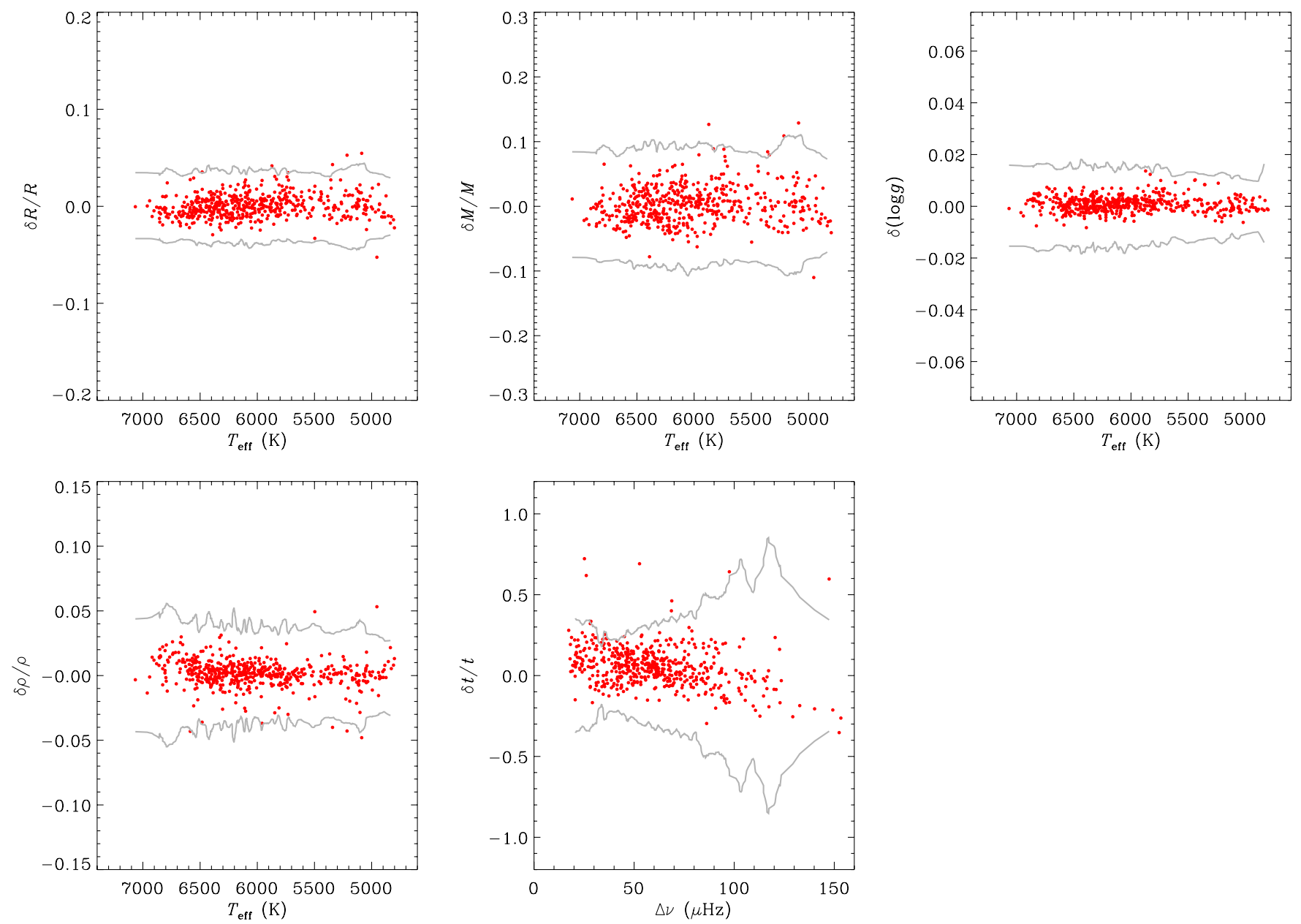

Figure 6. Fractional differences in estimated stellar properties returned by the BeSPP pipeline (run in scaling mode) and the YB pipeline, but with both coupled to the same YY grid. Results shown for the entire ensemble, with $\Delta v$ and $v_{\max }$, the photometric (IRFM) $T_{\text {eff }}$ and field [Fe/H] values used as inputs). Plot style as per Figure 4.

(A color version of this figure is available in the online journal.)

RADIUS follows a slightly different approach. It finds all models whose parameters lie within $3 \sigma$ of the observations. Properties are estimated from the properties of the most likely model, with the $1 \sigma$ uncertainties estimated as one-sixth of the maximum range of the selected models.

SEEK compares an observed star with every model of the grid and makes a probabilistic assessment of the stellar properties with the help of Bayesian statistics. Each stellar model in the grid is assigned a posterior probability that is the product of a Gaussian likelihood for each observable and an appropriate prior for the desired parameters. The probabilities are normalized so that the sum over all the stellar models is unity. For a given property, the probabilities are then summed in a suitable range of bins. In effect, one constructs a histogram of the desired property where each stellar model is weighted by its posterior probability. By associating the center of each bin with its height, a probability density function is created from which the final values of the properties are derived. The priors used are flat for age, metallicity, initial helium ratio, and mixing length parameter. The only non-flat prior is that of the initial mass function. SEEK used $v_{\max }$ only to select models, but does not use this parameter to obtain the final result.

The GOE pipeline is an independent implementation of the SEEK method. While the Bayesian method defined by the SEEK algorithm allows for the inclusion of different types of prior information, the Göttingen implementation only includes priors that correct for the non-uniform distribution of models in metallicity and age. To correct the age distribution, each model is weighted by the time-step of that model, so that models that are evolving more slowly are more likely. This counteracts the fact that evolutionary codes calculate more models in rapid phases of evolution. Without this correction, the results would be biased toward these rapid phases.

The YB pipeline was used with 5 different grids - the models from the Dartmouth group (Dotter et al. 2008), those of the Padova group (Marigo et al. 2008; Girardi et al. 2000), the models that comprise the Yonsei-Yale (YY) isochrones (Demarque et al. 2004), a grid of models constructed with the Yale Stellar Evolution Code (YREC; Demarque et al. 2008) and described by Gai et al. (2011) (we refer to this set as YREC), and another set of models constructed with YREC (we refer to this grid as YREC2) that has been described by Basu et al. (2012).

Although the YREC and YREC2 grids were constructed with the same code, they have different physics. These grids were calculated using different nuclear reaction rates, different relative heavy-element abundances and a different helium enrichment law. Additionally, all models in the YREC grid were calculated with the same value of the mixing-length parameter; YREC2 on the other hand consists of five sub-grids, each sub-grid constructed with a different value of the mixing-length parameter. 

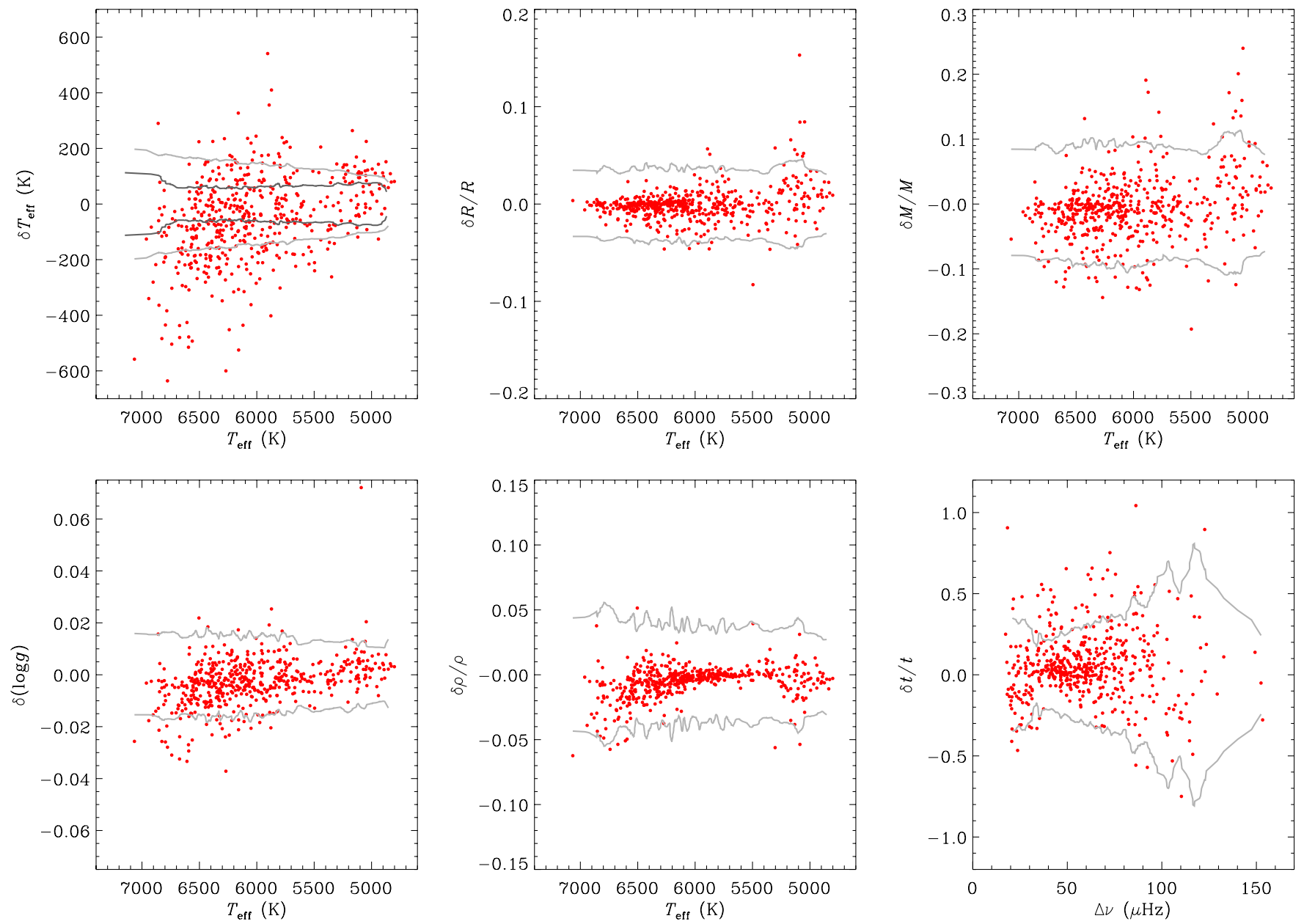

Figure 7. Fractional differences in estimated properties returned by the BeSPP pipeline run with the GARSTEC grid (run using model-calculated eigenfrequencies to estimate the $\Delta v$ of each model in the grid) for analyses performed on the entire ensemble with different $T_{\text {eff }}$ as inputs. Differences are plotted in the sense: results with SDSS-calibrated $T_{\text {eff }}$ minus results with IRFM-calculated $T_{\text {eff }}$. Gray lines mark the median $1 \sigma$ envelope (over all pipelines) of the returned, formal uncertainties. The top left-hand panel shows the absolute temperature differences (same sense), with the gray lines following the median $1 \sigma$ envelope of the IRFM uncertainties, and the black lines the envelope of the SDSS-calibrated uncertainties.

(A color version of this figure is available in the online journal.)

Table 3

Grid-based Pipelines and the Grids of Models

\begin{tabular}{|c|c|c|c|c|c|c|c|c|c|c|}
\hline Pipeline & Grid & $\begin{array}{l}\text { Mixing } \\
\text { Length } \alpha\end{array}$ & $\begin{array}{l}\text { Definition } \\
{[\mathrm{Fe} / \mathrm{H}]=0}\end{array}$ & Diffusion & $\begin{array}{c}\text { Core } \\
\text { Overshoot }\end{array}$ & $Y_{0}$ & $\begin{array}{l}\text { Enrichment } \\
\quad \text { Law }\end{array}$ & $\begin{array}{l}\text { Reaction } \\
\text { Rates }\end{array}$ & $\begin{array}{l}\text { High/low-T Z-Mixture } \\
\text { Opacities }\end{array}$ & EOS \\
\hline YB & Marigo & 1.680 & $Z=0.019$ & None & Func. of $M$ & Variable & $Y=0.23+2.25 Z$ & CF88/LP90 & OPAL/AF GN93 & AHD/ST \\
\hline YB & YREC & 1.826 & $Z / X=0.023$ & $Y, Z$ & $0.2 H_{p}$ & Variable $^{(3)}$ & $\Delta Y / \Delta Z=1$ & NACRE & OPAL/F05 GS98 & OPAL05 \\
\hline YB & YREC2 & $1.4-2.2$ & $Z / X=0.023$ & None & $0.2 H_{p}$ & Variable & $Y_{0}=0.245+1.54 Z_{0}$ & A98 $8^{(4)}$ & OPAL/F05 AGSS09 & OPAL05 \\
\hline BeSPP & GARSTEC & 1.81 & $Z / X=0.0229$ & $Y, Z$ & Diffusive $^{(5)}$ & Variable $^{(6)}$ & $Y_{0}=Y_{i}+1.4\left(Z-Z_{i}\right)$ & A11 & OPAL/F05 GS98 & $\mathrm{C} 03$ \\
\hline BeSPP & BASTI & 1.913 & $Z / X=0.0245$ & None & None & Variable $^{(7)}$ & $\Delta Y / \Delta Z=1.4$ & $\mathrm{NACRE}^{(8)}$ & OPAL/AF GN93 & $\mathrm{C} 03$ \\
\hline SEEK & ASTEC 3 & $0.8-2.3$ & $Z / X=0.0229$ & None & None & Variable $^{(11)}$ & $\cdots$ & BP95 & OPAL/AF GN93 & OPAL96 \\
\hline Göttingen (GOE) & CESAM2k & $0.75-1.75$ & $Z=0.0122$ & None & None & $Y_{0}=0.2607^{(12)}$ & None & $\mathrm{NACRE}^{(4)}$ & OPAL/F05 AGS05 & OPAL05 \\
\hline
\end{tabular}

Notes. (1) Except $\mathrm{N}(p, \gamma) \mathrm{O}, 3 \alpha, \mathrm{C}(\alpha, \gamma) \mathrm{O}$; (2) ideal EOS with Debye-Huckle correction $\left(M \geqslant 0.8 M_{\odot}\right)$; (3) $Y_{0}=0.246$ at $Z_{0}=0.01695$; (4) except for $\mathrm{N}(p, \gamma) \mathrm{O}$; (5) diffusive $(f=0.016)$ but restricted to smaller values for small convective cores; (6) $Z_{i}=0.01876, Y_{i}=0.26896$; (7) $Y_{0}=245$ at $Z_{0}=0.0001 ;(8)$ except for $\mathrm{C}(\alpha, \gamma) \mathrm{O}$; (9) $X_{i}$ kept constant at 0.71 for different $Z_{i}$; (10) $X_{i}$ kept constant at $0.7, Z_{i}$ varies; (11) different combinations of $Z_{i}$ and $X_{i}$. Many $X_{i}$ for same $Z_{i}$; (12) $Z / Z_{\odot}$ from 0.5 to 2.0 , where $Z_{\odot}=0.13757$; masses from $0.75 M_{\odot}$ to $1.75 M_{\odot}$. A98: Adelberger et al. (1998); A11: Adelberger et al. (2011); AF94: Alexander \& Ferguson (1994); AGS05: Asplund et al. (2005); AGSS09: Asplund et al. (2009); B89: Bahcall (1989); BP95: Bahcall \& Pinsonneault (1992); CF88: Caughlan \& Fowler (1988); C03: Cassisi et al. (2003); E73: Eggleton et al. (1973); F05: Ferguson et al. (2005); G11: Gai et al. (2011); GN93: Grevesse \& Noels (1993); GS98: Grevesse \& Sauval (1998); K91: Kurucz (1991); LP90: Landré et al. (1990); MHD: Mihalas et al. (1988); ST88: Straniero (1988). 

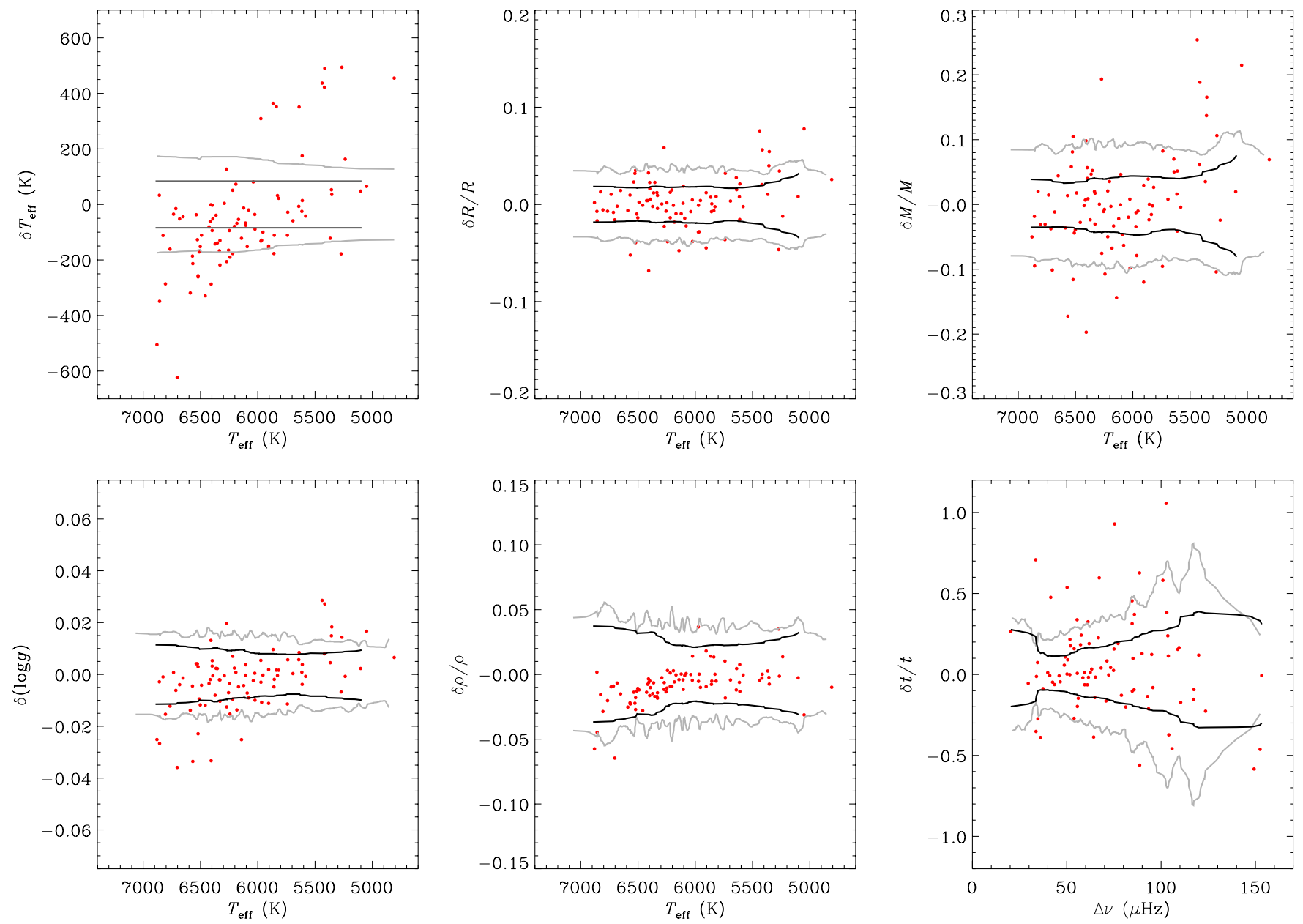

Figure 8. Fractional differences in estimated properties returned by the BeSPP pipeline run with the GARSTEC grid, for analyses performed on the subset of stars with spectroscopic $T_{\text {eff }}$ and $[\mathrm{Fe} / \mathrm{H}]$ available. Differences are plotted in the sense: results with spectroscopic $T_{\text {eff }}$ and $[\mathrm{Fe} / \mathrm{H}]$ minus results with IRFM $T_{\text {eff }}$ and field-average $[\mathrm{Fe} / \mathrm{H}]$. Gray lines mark the median $1 \sigma$ envelope (over all pipelines) of the returned, formal uncertainties of the IRFM-based results, the black lines the median envelopes given by the spectroscopic-based results. The top left-hand panel shows the absolute temperature differences (same sense), with the gray lines following the median $1 \sigma$ envelope of the IRFM uncertainties, and the black lines the envelope of the Bruntt et al. (2012) spectroscopic $T_{\text {eff }}$ uncertainties.

(A color version of this figure is available in the online journal.)

For all models in these grids, the seismic parameter $\Delta v$ was calculated using the scaling relation given in Equation (1).

The BeSPP pipeline was run with two grids. The first grid comprises models constructed with the GARSTEC code (Weiss \& Schlattl 2008) and the parameters of the grid are described in Silva Aguirre et al. (2012). The $\Delta v$ of each model in this grid was determined using the calculated frequencies of each model (one set of results) and also using the scaling relation in Equation (1) (to give a second set of results that we call BeSPPscale). The second grid of models are the BASTI models of Pietrinferni et al. (2004) computed specifically for use in asteroseismic studies, and as described in Silva Aguirre et al (2013). In this case $\Delta v$ for the models was calculated using only the scaling relation.

RadEx 10, RADIUS and SEEK used models constructed with the ASTEC code (Christensen-Dalsgaard 2008). The models used in RadEx10, referred to as ASTEC1, are described by Creevey et al. (2013). $\Delta v$ for this grid was calculated from the scaling relation. The models for RADIUS are described in Stello et al. (2009) and Creevey et al. (2012), and these models are henceforth referred to as ASTEC2. While $\Delta v$ for this grid was calculated using the scaling relations, it was also calculated for a subset of ASTEC2 using individual frequencies. The models used by SEEK are referred to as ASTEC3. For this grid $\Delta v$ was calculated using eigenfrequencies. Although all the grids were constructed with the same code, they have different physics, such as low-temperature opacities and equation of state, and different input parameters.

GOE was run on a grid calculated with the CESTAM code (Marques et al. 2013), which is derived from the CESAM2k code described in Morel \& Lebreton (2008). The $\Delta v$ of each model was calculated from the eigenfrequencies.

The parameters of the different grids of models are listed in Table 3. As can be seen, the grids are diverse, not only constructed with different codes, but also with different input physics. For example, there are grids of models with and without diffusion and overshoot; and also grids constructed with different model prescriptions, e.g., for overshoot and $\mathrm{He}$ enrichment.

\section{COMPARISON OF RESULTS FROM DIFFERENT GRID-PIPELINE COMBINATIONS}

Before consolidating the results to give tables of stellar properties, we first present a comparison of the estimates returned by the various grid-pipelines. To frame the discussion we have selected representative plots of differences shown by 

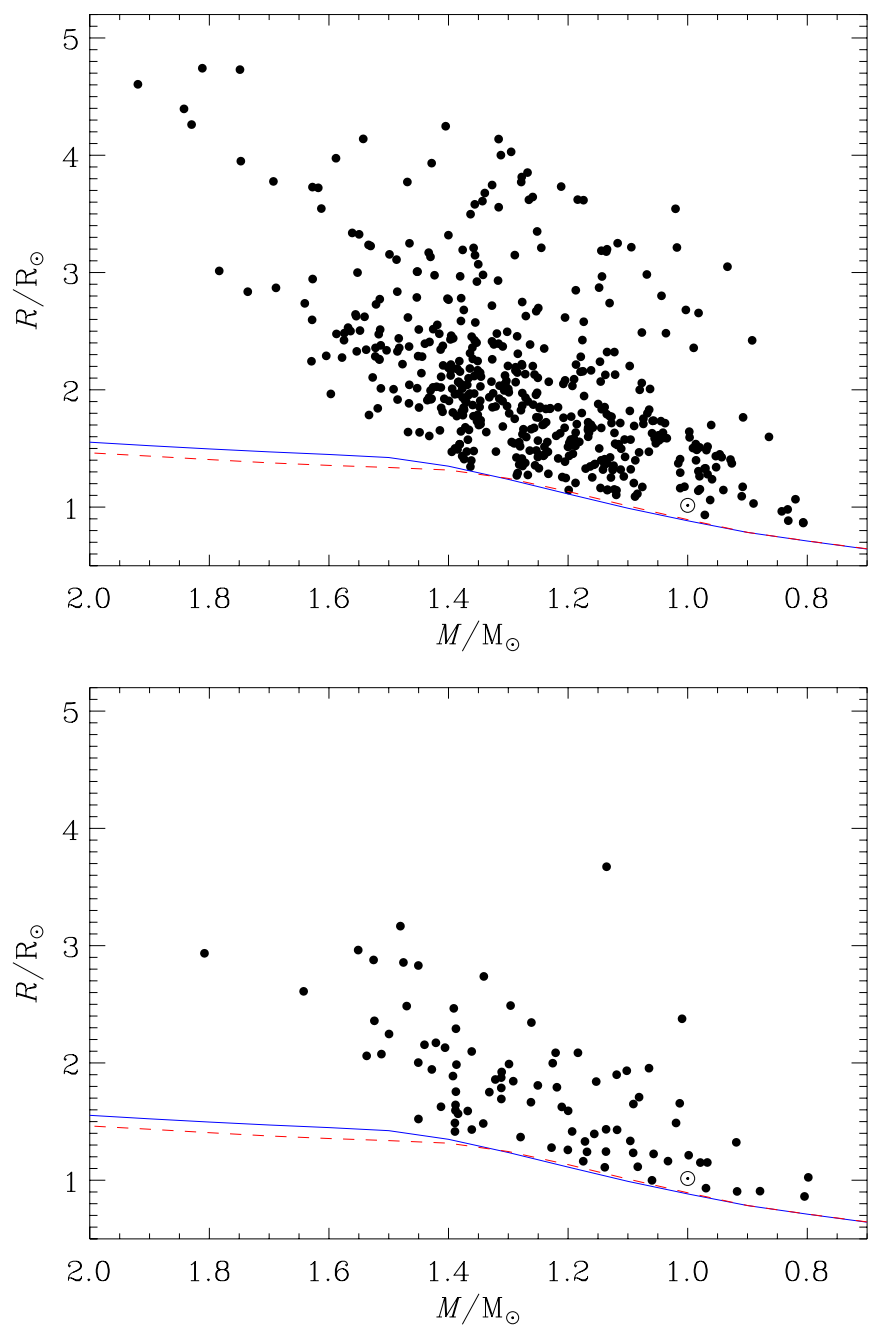

Figure 9. Estimated masses and radii for the full cohort with IRFM inputs (top panel) and the smaller cohort with spectroscopic inputs (bottom panel). The solid and dashed lines mark the ZAMS for $[\mathrm{Fe} / \mathrm{H}]=0$ and -0.2 , respectively (computed using the YREC code).

(A color version of this figure is available in the online journal.)

certain grid-pipeline combinations. Appendix A shows detailed plots (Figures 13 through 22) of differences for all the pipelines.

We begin by presenting results given by the BeSPP pipeline coupled to the GARSTEC grid. The BeSPP/GARSTEC combination can be run in two ways, one where $\Delta v$ is calculated using the eigenfrequencies, and one where $\Delta v$ is calculated using the scaling relations. This allows us to test the differences in the results caused by how $\Delta v$ is calculated, independently of differences in results arising from the analysis pipeline or the grid of stellar models used.

Figure 4 shows the resulting differences in estimated stellar properties for the entire ensemble, with $\Delta v$ and $v_{\max }$, the photometric (IRFM) $T_{\text {eff }}$ and field $[\mathrm{Fe} / \mathrm{H}]$ values used as inputs. The plots show differences in the sense scaling-mode outputs minus frequency-mode outputs, and are fractional differences in $R, M, \rho$ and age $t$; and absolute differences in $\log g$. Age differences have been plotted against $\Delta v$ to delineate approximately the evolutionary state (since $\Delta v$ gives a first-order discrimination of main-sequence, sub-giant and low-luminosity red-giant targets). The gray lines mark envelopes corresponding to the median of the formal $1 \sigma$ uncertainties returned by all gridpipelines. Medians were calculated in 10-target batches sorted on the independent variable used for the plots ( $T_{\text {eff }}$ for $R, M$, $\log g$ and $\rho$; and $\Delta v$ for $t$ ). These lines are included to help judge the typical precision only; the uncertainties in the results of any particular star may be slightly different.

We see clearly the impact of adopting the scaling relation to compute $\Delta v$ instead of using model-calculated eigenfrequencies. The "boomerang" shaped trends arise directly from the similar-shaped differences shown between $\Delta v$ calculated using the scaling relation and the individual eigenfrequencies, as discussed earlier in Section 2.2. The impact is strongest in the estimated densities. The boomerang shape is absent from the age differences, although there is a small positive bias arising from the negative differences displayed in the masses. It is worth noting that, at the level of precision of these data, the boomerang-shaped differences lie largely within the median $1 \sigma$ uncertainty envelopes.

In addition to the impact of the scaling relation, we also expect differences in results due to the choice of grid of stellar evolutionary models, and the actual pipelines themselves, i.e., due to differences in methodology and procedure. First, Figure 5 shows a representative example of changing grids. Here, we plot differences between BeSPP/BASTI (scaling-mode) and BeSPP/ GARSTEC (frequency-mode). The boomerang-shaped trends from Figure 4 are still present, but there is now increased scatter due to differences between the grids, i.e., model dependencies in the results. This increased level of scatter is also present in differences between other grid-pipeline combinations (see plots in Appendix A). Next, we isolate the impact of scatter due to different fitting methodologies by coupling different pipelines to the same grid of models. Figure 6 shows a representative example, where we coupled the BeSPP pipeline to the YY grid in order to calculate the plotted differences, between BeSPP/YY and YB/YY. Although the differences lie fairly comfortably within the formal uncertainty envelopes, they are not entirely negligible (note how the ages show a small systematic bias in $\Delta v)$. Tests of the grid-based and pipeline-based errors, using other grid-pipeline combinations at our disposal, indicate that differences given by the choice of grid are typically more important than those given by the pipeline code. Our consolidation of results to give final uncertainties on the stellar properties includes the effects of both error contributions (see Section 6). Again, we stress that on the whole these combined differences lie within the median $1 \sigma$ uncertainty envelopes.

It is not surprising that of all the properties, the ages for the full cohort are the most scattered and poorly constrained. They also have the largest formal fractional uncertainties. Neither $\Delta v$ nor $v_{\max }$ contain any explicit dependence on age; and for the full cohort we lack strong constraints on the metallicities, which determine how fast a star evolves and also the effective temperature for a given luminosity. We see clear evidence of the uncertainties dropping in more evolved stars, i.e., the lower $\Delta v$ stars that have evolved off the main-sequence (see the median formal uncertainty envelopes in the plots). This is consistent with the results found by Gai et al. (2011). The reason for the lower uncertainty is easy to understand. The subgiant phase is rapid and $\Delta v$ and $v_{\text {max }}$, as well as $T_{\text {eff }}$, change much more rapidly than on the main sequence, thereby giving a better determination of the age for stars in this phase of evolution. Use of individual frequencies, or small frequency separations involving dipole $(l=1)$ and quadrupole $(l=2)$ modes, would lead to a considerable tightening of the age uncertainties of the mainsequence stars (see, e.g., Christensen-Dalsgaard 1993; Cunha et al. 2007; Chaplin \& Miglio 2013; and references therein). 


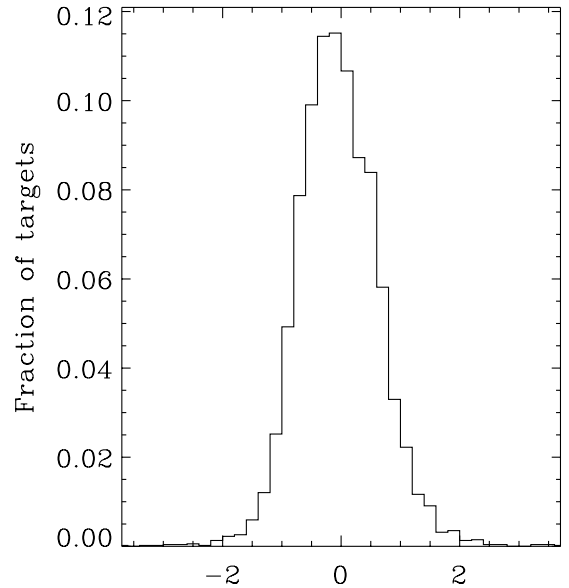

Normalized $R$ residual (sigma)

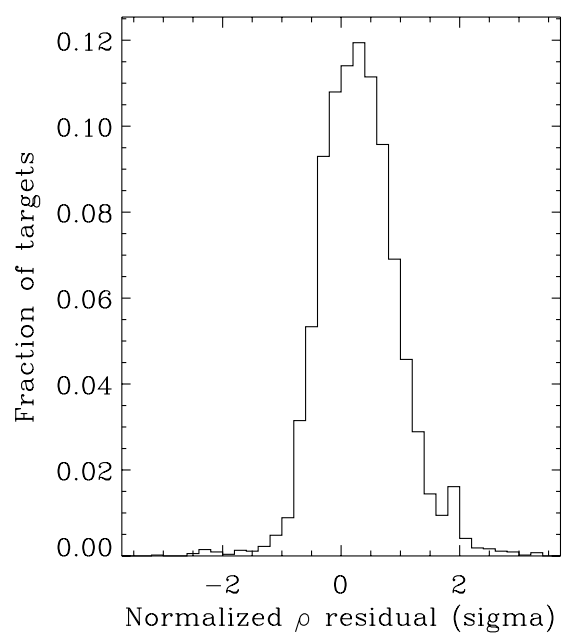

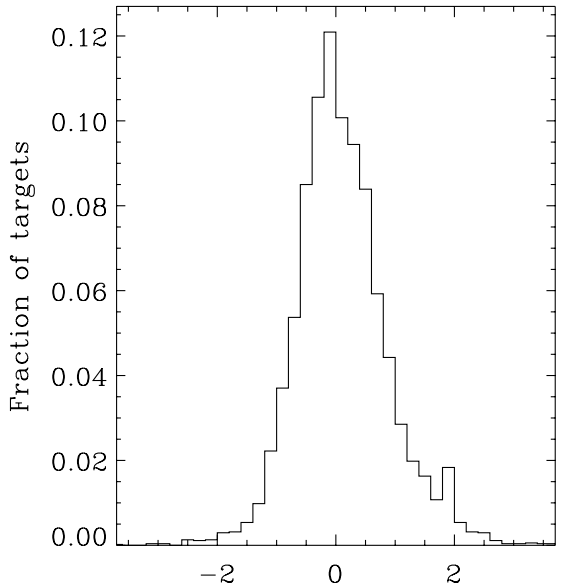

Normalized $M$ residual (sigma)

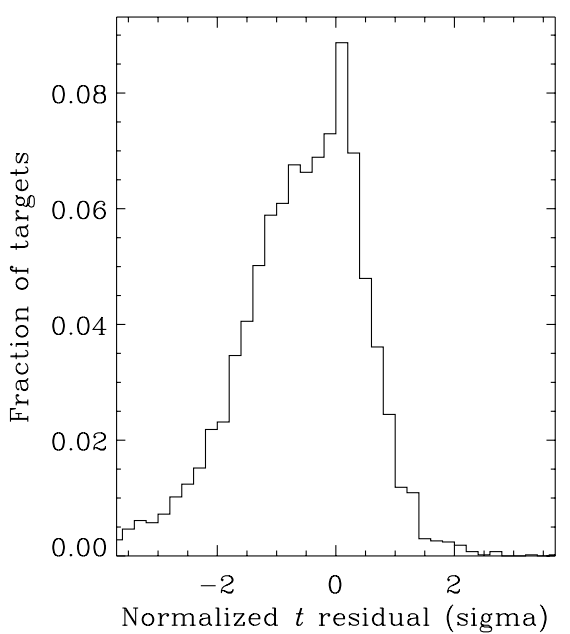

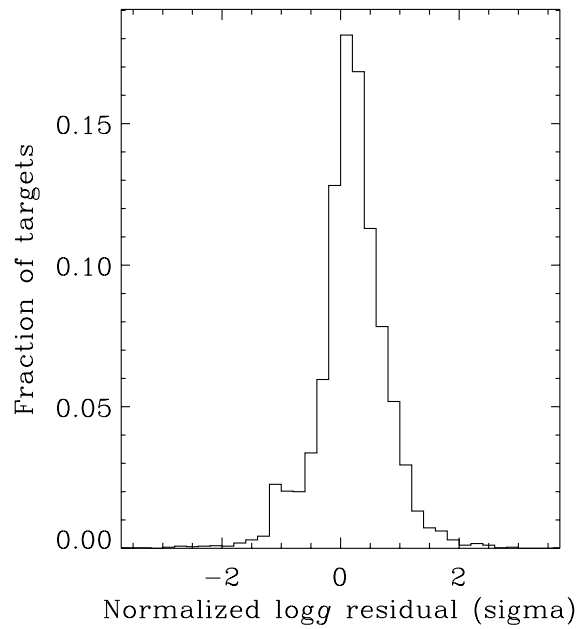

Normalized $\log g$ residual (sigma)

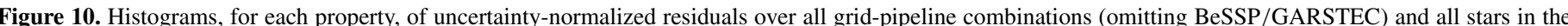
IRFM cohort (see text). Residuals calculated with respect to the BeSSP/GARSTEC results run in frequency mode.

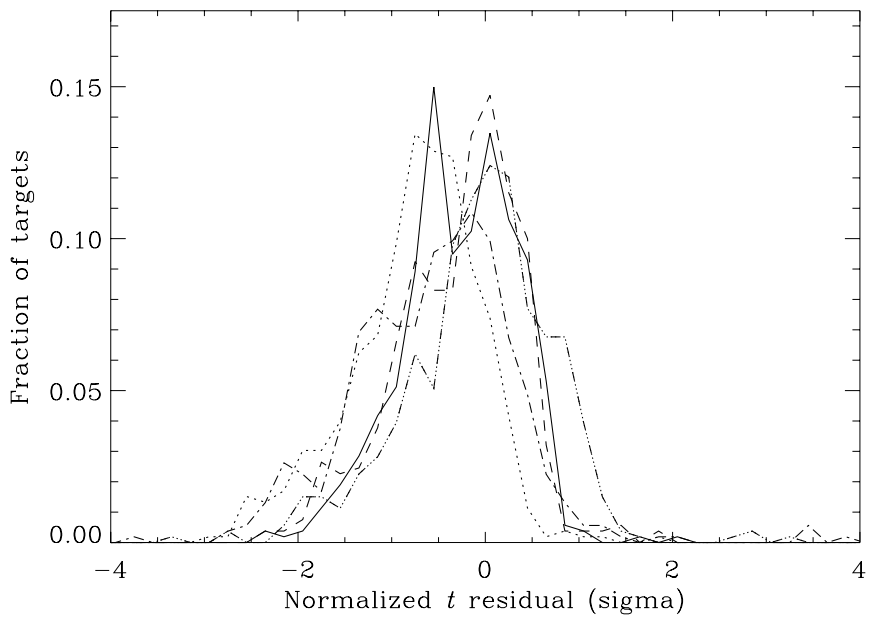

Figure 11. Histogram for estimated ages, in normalized residual form as per the age histogram in Figure 10, but showing results for each of coupled to the YB pipeline. Residuals again calculated with respect to the BeSSP/GARSTEC results run in frequency mode. Note that for clarity we have plotted each of the histograms by joining the midpoints of the bins.

The results for the subset of stars having complementary spectroscopic inputs are also encouraging (see Appendix A for detailed plots). Superior constraints on $[\mathrm{Fe} / \mathrm{H}]$ and $T_{\text {eff }}$ translate, as expected, to higher precision in the estimated

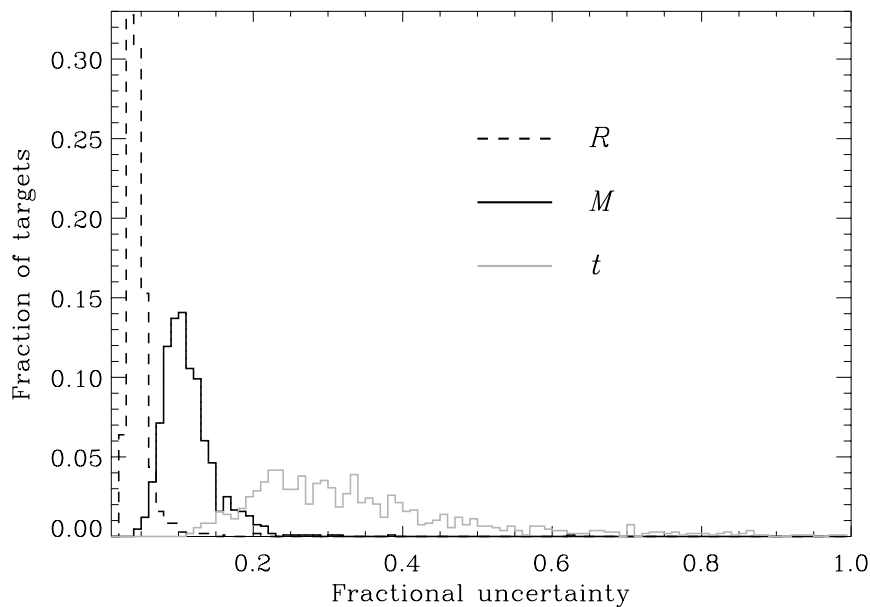

Figure 12. Histograms of fractional uncertainties for estimated radii $R$, masses $M$, and ages $t$, of the full cohort of stars (using input effective temperatures from the IRFM set; see figure legend).

properties. There is also a slightly higher fraction of differences lying outside the median $1 \sigma$ error envelopes. Nevertheless, consistency between the pipelines remains good. The scatter between pipelines is again, not surprisingly, largest for the age 

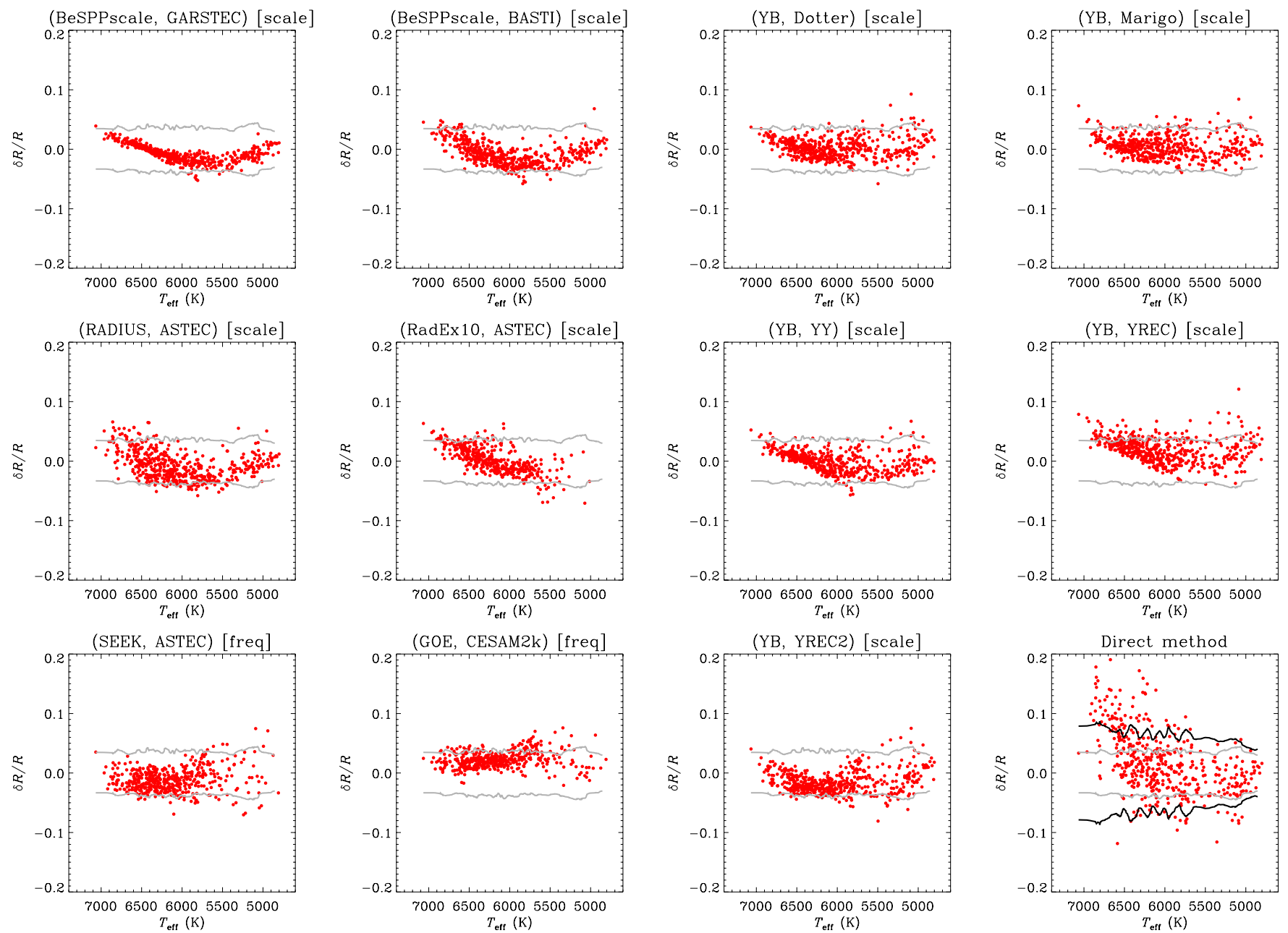

Figure 13. Fractional differences in estimated radii, $R$, for analyses performed on the entire ensemble, with $\Delta v$ and $v_{\max }$, the photometric (IRFM) $T_{\text {eff }}$ and field [Fe/H] values used as inputs. The plots show differences with respect to the BeSPP pipeline run with the GARSTEC grid run using model-calculated eigenfrequencies to estimate the $\Delta v$ of each model in its grid. Gray lines mark the median $1 \sigma$ envelope of the grid-pipeline returned, formal uncertainties. These lines are included to help judge the typical precision only. The bottom right-hand panel shows results from direct application of the scaling relations, the black lines showing the median $1 \sigma$ envelope on the resulting uncertainties.

(A color version of this figure is available in the online journal.)

estimates, where the reduction in the input errors has brought the model-dependencies of stellar age estimates to the fore.

We also checked the impact on the results of omitting $v_{\max }$ from the input data (i.e., using $\Delta v$ as the only seismic input), both for the same pipeline coupled to different grids, and different pipelines coupled to the same grid. We find that changes to the estimated properties - i.e., differences between properties given by $\Delta v$ and $v_{\max }$, and $\Delta v$ alone - are less than $1 \sigma$ for most of the stars. These differences are found to be largely due to differences in the grids, not the pipelines.

Another source that can contribute to differences in the estimated properties is the input set of effective temperatures, $T_{\text {eff }}$. Note that in Section 6 we provide estimated stellar properties for each set of input $T_{\text {eff }}$.

Figure 7 shows the impact on the full-ensemble results of switching from one set of input photometric $T_{\text {eff }}$ to the other. The top left-hand panel shows SDSS-calibrated $T_{\text {eff }}$ minus the IRFM-calculated $T_{\text {eff }}$. The gray lines follow the median $1 \sigma$ envelope of the IRFM uncertainties, and the black lines the envelope of the SDSS-calibrated uncertainties. The other panels plot the fractional differences in estimated properties returned by the BeSPP pipeline, with differences plotted in the sense SDSS minus IRFM. As in the previous figures, gray lines mark the median $1 \sigma$ envelopes (over all pipelines) of the returned, formal uncertainties from the IRFM results. Differences between the two sets of temperatures may have some of their origin in differences in the adopted reddening: Pinsonneault et al. used the reddening information in the KIC to derive the SDSS temperatures, whilst we used the reddening maps of Drimmel et al. (2003) to derive the IRFM temperatures.

We may use the simple scaling relations to help us understand the trends revealed in Figure 7 . The relations imply that $M \propto T_{\text {eff }}^{1.5}, R \propto T_{\text {eff }}^{0.5}$ and hence $g \propto T_{\text {eff }}^{0.5}$ (all other things being equal). The trend in the $T_{\text {eff }}$ is such that the SDSS temperatures are on average slightly lower than the IRFM temperatures, most notably at high $T_{\text {eff }}$, whilst the differences are slightly reversed at lower $T_{\text {eff }}$. This trend seems to be reflected in the plotted property differences (most notably in the masses), which show a small negative average bias. The differences again fall largely within the $1 \sigma$ uncertainty envelopes.

Figure 8 shows the impact of switching the input data from the photometric to the spectroscopic $T_{\text {eff }}$ and $[\mathrm{Fe} / \mathrm{H}]$. The top left-hand panel shows the spectroscopic $T_{\text {eff }}$ minus the IRFM-calculated $T_{\text {eff }}$. The gray lines follow the median 

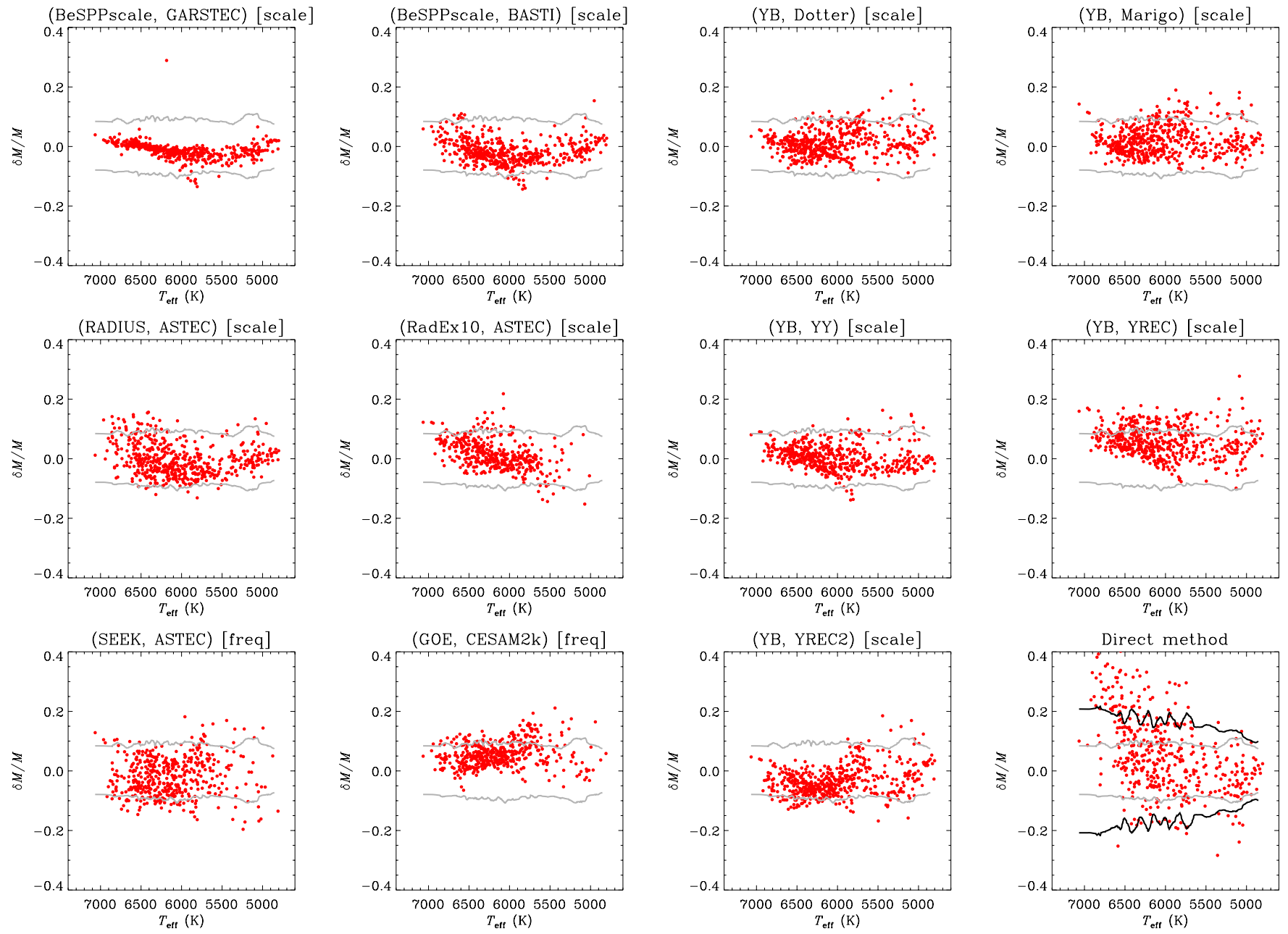

Figure 14. As per Figure 13, but for fractional differences in mass $M$.

(A color version of this figure is available in the online journal.)

Table 4

Estimated Stellar Properties Using SDSS-calibrated $T_{\text {eff }}$ and Field-average $[\mathrm{Fe} / \mathrm{H}]$ Values

\begin{tabular}{lccccc}
\hline \hline KIC & $\begin{array}{c}M \\
\left(M_{\odot}\right)\end{array}$ & $\begin{array}{c}R \\
\left(R_{\odot}\right)\end{array}$ & $\begin{array}{c}\rho \\
\left(\rho_{\odot}\right)\end{array}$ & $\begin{array}{c}\log g \\
(\mathrm{dex})\end{array}$ \\
\hline 1430163 & $1.38+0.14-0.10$ & $1.49+0.05-0.05$ & $0.4195+0.0189-0.0181$ & $4.234+0.014-0.015$ & $1.3+0.6-0.7$ \\
1435467 & $1.16+0.20-0.06$ & $1.63+0.08-0.05$ & $0.2732+0.0073-0.0071$ & $4.088+0.019-0.014$ & $4.7+0.7-1.7$ \\
1725815 & $1.43+0.15-0.11$ & $2.02+0.08-0.08$ & $0.1732+0.0077-0.0074$ & $3.982+0.014-0.014$ & $3.1+0.7-0.8$ \\
2010607 & $1.37+0.15-0.14$ & $2.42+0.12-0.11$ & $0.0972+0.0079-0.0075$ & $3.809+0.025-0.025$ & $3.8+0.8-0.8$ \\
2309595 & $1.14+0.19-0.22$ & $2.40+0.19-0.23$ & $0.0828+0.0078-0.0067$ & $3.734+0.013-0.013$ & $6.0+4.5-1.5$ \\
2450729 & $1.11+0.12-0.13$ & $1.76+0.07-0.07$ & $0.2017+0.0054-0.0052$ & $3.988+0.017-0.017$ & $6.2+1.8-1.2$ \\
2837475 & $1.42+0.14-0.10$ & $1.63+0.06-0.05$ & $0.3286+0.0107-0.0107$ & $4.168+0.012-0.012$ & $1.9+0.4-0.7$ \\
2849125 & $1.38+0.29-0.12$ & $2.42+0.18-0.10$ & $0.0975+0.0061-0.0058$ & $3.814+0.016-0.016$ & $4.0+0.7-1.6$ \\
2852862 & $1.49+0.13-0.15$ & $2.10+0.07-0.08$ & $0.1609+0.0043-0.0042$ & $3.965+0.012-0.013$ & $3.0+0.7-0.7$ \\
2865774 & $1.28+0.11-0.21$ & $1.79+0.08-0.11$ & $0.2202+0.0165-0.0161$ & $4.030+0.022-0.025$ & $4.5+2.0-0.8$ \\
\hline
\end{tabular}

(This table is available in its entirety in a machine-readable form in the online journal. A portion is shown here for guidance regarding its form and content.)

$1 \sigma$ envelope of the IRFM uncertainties, and the black lines mark the envelope of the spectroscopic uncertainties. The other panels plot the fractional property differences returned by BeSPP (sense spectroscopic minus IRFM). Gray lines mark the median uncertainty envelopes from the IRFM-based results, the black lines the median envelopes given by the spectroscopic based-results. The trend in the temperature differences is quite similar to that shown in Figure 7, and the plots of the property differences again show a small negative bias, as expected. Not surprisingly, most differences lie well within the IRFM-based uncertainties (which we recall used the poorly constrained fieldaverage $[\mathrm{Fe} / \mathrm{H}])$.

Finally in this section, we note that Mosser et al. (2013) have recently discussed modifying the observed average $\Delta v$, for use with the scaling relations, to the value expected in the high-frequency asymptotic limit. The solar reference $\Delta v$ must also be modified. We have tested the impact on our results of applying this procedure, and find that it has a negligible 

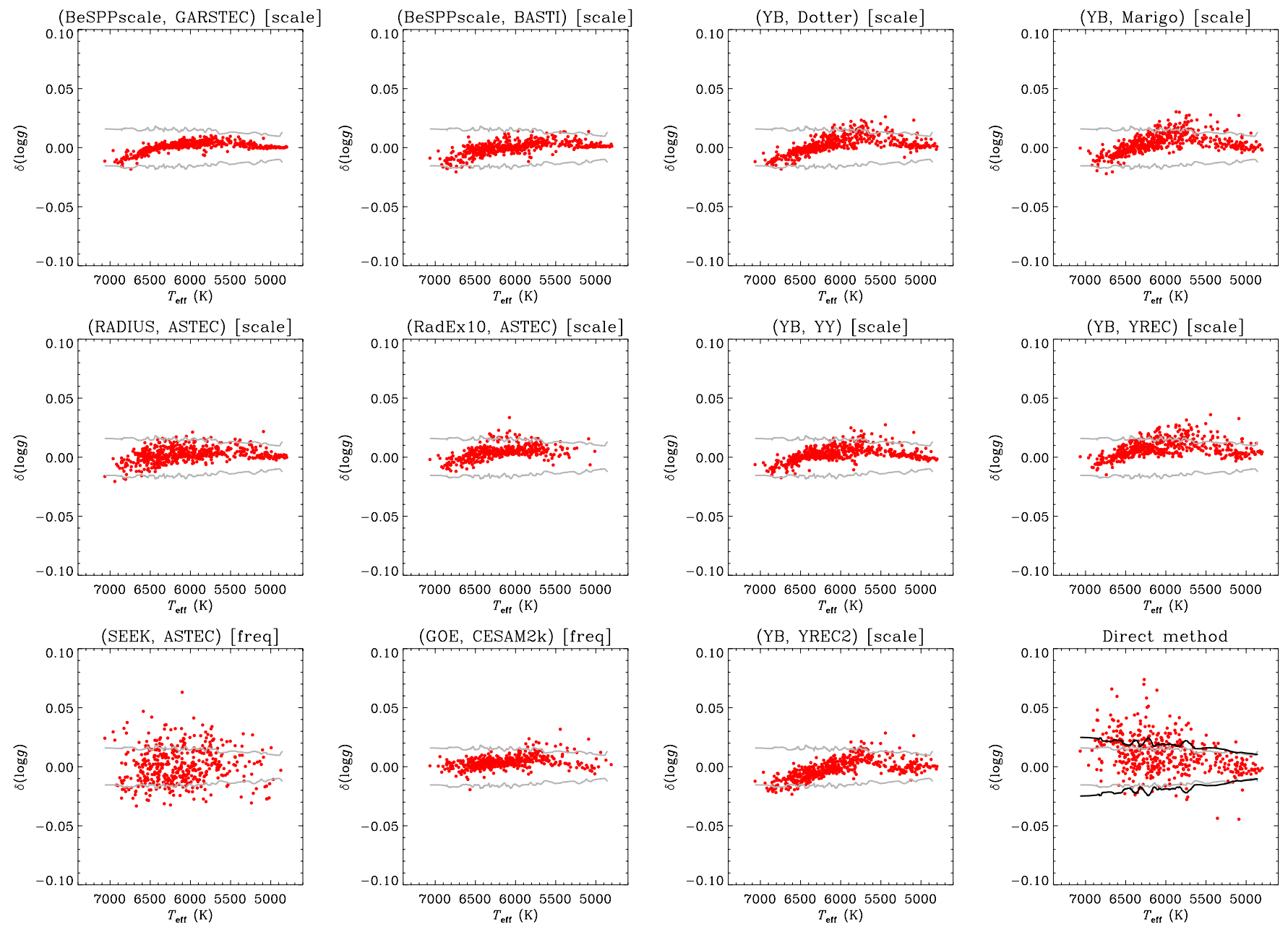

Figure 15. As per Figure 13, but for differences in $\log g$.

(A color version of this figure is available in the online journal.)

Table 5

Estimated Stellar Properties Using IRFM $T_{\text {eff }}$ and Field-average [Fe/H] Values

\begin{tabular}{lccccc}
\hline \hline KIC & $M$ & $R$ & $\rho$ & $t$ \\
$\left(\rho_{\odot}\right)$ & $\begin{array}{c}\log g \\
(\mathrm{dex})\end{array}$ & $\begin{array}{c}t \\
(\mathrm{Gyr})\end{array}$ \\
\hline 1430163 & $1.38+0.15-0.09$ & $1.49+0.05-0.05$ & $0.4219+0.0199-0.0198$ & $4.237+0.017-0.017$ & $1.3+0.6-0.9$ \\
1435467 & $1.19+0.20-0.08$ & $1.64+0.08-0.05$ & $0.2750+0.0082-0.0081$ & $4.091+0.022-0.016$ & $4.5+0.8-1.7$ \\
1725815 & $1.43+0.15-0.13$ & $2.02+0.08-0.08$ & $0.1733+0.0081-0.0077$ & $3.982+0.016-0.016$ & $3.2+0.9-0.9$ \\
2010607 & $1.48+0.15-0.14$ & $2.44+0.12-0.11$ & $0.1022+0.0086-0.0083$ & $3.835+0.027-0.028$ & $3.1+0.8-0.8$ \\
2309595 & $1.18+0.19-0.22$ & $2.42+0.18-0.21$ & $0.0825+0.0072-0.0063$ & $3.737+0.013-0.014$ & $5.5+3.8-1.2$ \\
2450729 & $1.13+0.11-0.13$ & $1.77+0.06-0.07$ & $0.2026+0.0055-0.0054$ & $3.992+0.018-0.017$ & $5.8+1.9-1.2$ \\
2837475 & $1.47+0.15-0.13$ & $1.64+0.06-0.06$ & $0.3325+0.0134-0.0127$ & $4.173+0.020-0.016$ & $1.8+0.6-0.9$ \\
2849125 & $1.52+0.17-0.19$ & $2.47+0.15-0.14$ & $0.0988+0.0062-0.0060$ & $3.826+0.016-0.016$ & $2.9+1.2-0.8$ \\
2852862 & $1.53+0.12-0.16$ & $2.11+0.06-0.08$ & $0.1636+0.0061-0.0053$ & $3.973+0.017-0.015$ & $2.6+0.8-0.7$ \\
2865774 & $1.30+0.12-0.15$ & $1.80+0.08-0.09$ & $0.2213+0.0162-0.0157$ & $4.036+0.023-0.024$ & $4.3+1.2-1.2$
\end{tabular}

(This table is available in its entirety in a machine-readable form in the online journal. A portion is shown here for guidance regarding its form and content.)

impact on the properties estimated by those pipelines that use the scaling-relation-computed $\Delta v$ for the stellar models. The changes in mass and radius are typically less than $1 \%$. The reason for the insignificant changes is as follows. The average correction for solar type stars is (fractionally speaking) quite small, and is basically offset by the similar fractional increase in the solar reference $\Delta v$. While the fractional modification to $\Delta v$ is not the same for all stars, it is a fairly weak function of $v_{\max }$, and hence luminosity $L$ (the correction is proportional approximately to $v_{\max }{ }^{-0.21}$, and $v_{\max } \propto M T_{\text {eff }}^{3.5} / L^{-1}$ ). Thus, the modification does not significantly affect the results (see also Hekker et al. 2013).

\section{TABLES OF ASTEROSEISMICALLY INFERRED STELLAR PROPERTIES}

Tables 4, 5 and 6 list estimated stellar properties from our analyses. Each table gives properties for a different set of $T_{\text {eff }}$ and 

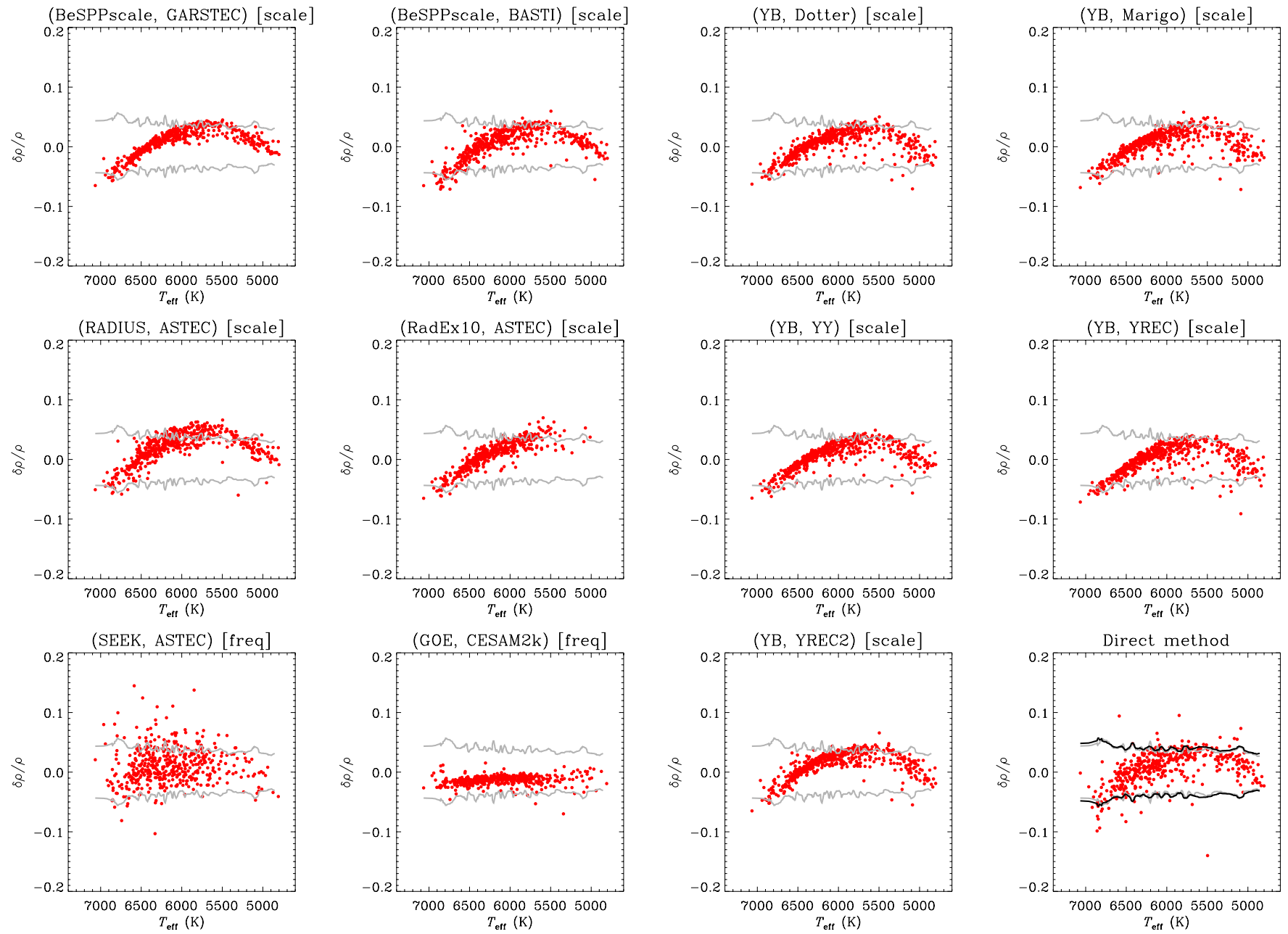

Figure 16. As per Figure 13, but for fractional differences in average density $\rho$.

(A color version of this figure is available in the online journal.)

Table 6

Estimated Stellar Properties Using Spectroscopic $T_{\text {eff }}$ and $[\mathrm{Fe} / \mathrm{H}]$ Values from Bruntt et al. (2012)

\begin{tabular}{lccccc}
\hline \hline KIC & $\begin{array}{c}M \\
\left(M_{\odot}\right)\end{array}$ & $\begin{array}{c}R \\
\left(R_{\odot}\right)\end{array}$ & $\begin{array}{c}\rho \\
\left(\rho_{\odot}\right)\end{array}$ & $\begin{array}{c}\log g \\
(\mathrm{dex})\end{array}$ \\
\hline 1430163 & $1.34+0.06-0.06$ & $1.48+0.03-0.03$ & $0.4099+0.0171-0.0174$ & $4.221+0.013-0.014$ & $1.9+0.6-0.5$ \\
1435467 & $1.31+0.08-0.16$ & $1.69+0.04-0.06$ & $0.2675+0.0066-0.0067$ & $4.094+0.012-0.019$ & $3.7+1.7-0.8$ \\
2837475 & $1.41+0.06-0.04$ & $1.63+0.03-0.03$ & $0.3295+0.0105-0.0103$ & $4.167+0.010-0.010$ & $2.0+0.3-0.4$ \\
3424541 & $1.39+0.07-0.06$ & $2.47+0.06-0.06$ & $0.0930+0.0046-0.0044$ & $3.797+0.016-0.015$ & $4.0+0.6-0.6$ \\
3427720 & $1.08+0.06-0.06$ & $1.12+0.02-0.02$ & $0.7817+0.0262-0.0255$ & $4.378+0.012-0.012$ & $3.5+1.5-1.4$ \\
3456181 & $1.36+0.07-0.12$ & $2.10+0.05-0.06$ & $0.1463+0.0042-0.0040$ & $3.924+0.010-0.012$ & $3.9+1.0-0.5$ \\
3632418 & $1.31+0.08-0.07$ & $1.88+0.04-0.04$ & $0.1993+0.0034-0.0034$ & $4.010+0.010-0.009$ & $4.1+0.5-0.6$ \\
3656476 & $1.10+0.05-0.06$ & $1.34+0.03-0.03$ & $0.4588+0.0109-0.0105$ & $4.225+0.008-0.008$ & $7.2+2.2-1.8$ \\
3733735 & $1.39+0.04-0.05$ & $1.42+0.03-0.03$ & $0.4883+0.0235-0.0231$ & $4.277+0.014-0.014$ & $0.8+0.4-0.4$ \\
4586099 & $1.32+0.08-0.11$ & $1.86+0.04-0.05$ & $0.2049+0.0064-0.0062$ & $4.018+0.012-0.013$ & $3.9+0.9-0.6$ \\
\hline
\end{tabular}

(This table is available in its entirety in a machine-readable form in the online journal. A portion is shown here for guidance regarding its form and content.)

$[\mathrm{Fe} / \mathrm{H}]$ inputs (SDSS-calibrated $T_{\text {eff }}$ and field-average $[\mathrm{Fe} / \mathrm{H}]$ values; IRFM $T_{\text {eff }}$ and field-average $[\mathrm{Fe} / \mathrm{H}]$ values; and Bruntt et al. spectroscopic values, respectively). Figure 9 plots results given by the IRFM inputs (top panel) and spectroscopic inputs (bottom panel) in the mass-radius plane, with the location of the ZAMS also plotted for solar and sub-solar $[\mathrm{Fe} / \mathrm{H}]$ (see caption).

These final properties come from coupling BeSPP to the GARSTEC grid, run in the mode where theoretical oscillation frequencies of each model were used to compute average model $\Delta v$. This meant that the final properties are not subject to the small bias in the $\Delta v$ scaling relation (manifested as the boomerang-shaped plot differences). Details of the adopted input physics for GARSTEC are given in Table 3.

The uncertainties for the properties of each star listed in Tables 4, 5 and 6 were given by adding (in quadrature) the uncertainty returned by the BeSPP pipeline to the standard 

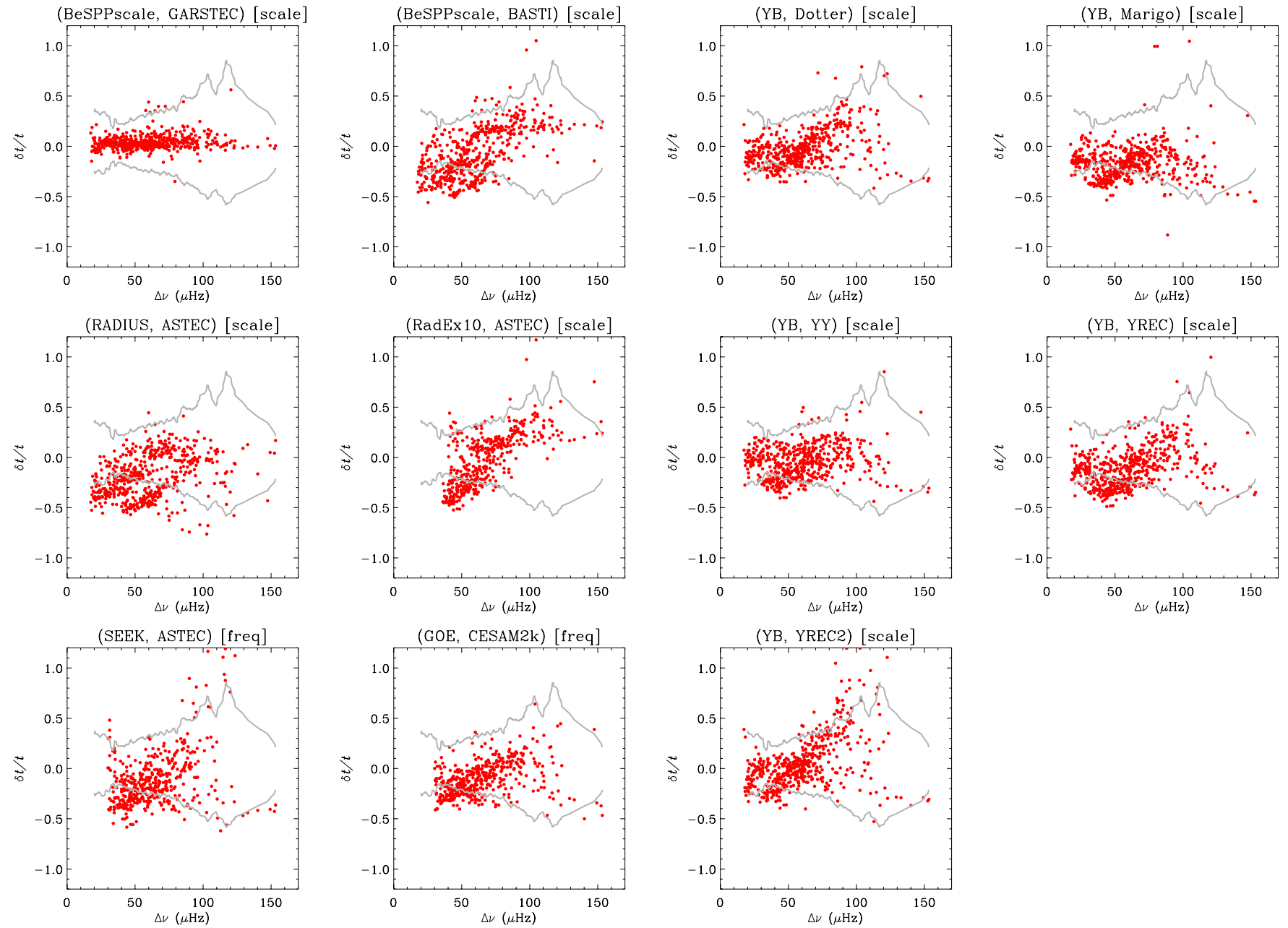

Figure 17. As per Figure 13, but for fractional differences in age, $t$, and with $\Delta v$ as the independent variable for the plot.

(A color version of this figure is available in the online journal.)

deviation (scatter) of the star's property over all grid-pipeline combinations. By including this scatter in the error budget we capture explicitly the uncertainties arising from differences between the commonly used grids of models we have adopted, and scatter due to different methodologies from the different pipeline codes.

Distributions of the scatter between pipelines are shown in Figure 10. The histograms, which show results for the IRFM results on the full cohort, were produced as follows. Omitting results from BeSSP/GARSTEC, for each star we computed residuals for each grid-pipeline with respect to the BeSSP/ GARSTEC result (frequency mode), normalizing each residual by the median property uncertainty given by the pipelines for that star. We then accumulated residuals for all stars in the cohort, and binned the residuals to give the plotted "super distributions."

The most striking aspect of the histograms is their Gaussianlike appearance (but see comments below on the slightly skewed age distribution). This indicates that when a wide selection of grids is used, differences in the input ingredients and physics give, to first order, normally distributed-like scatter. This lends weight to our approach of including this scatter contribution in our final error budget by using the standard deviation. Note that residuals for the gravities are the most peaked, possibly suggesting that the formal uncertainties in $\log g$ are slight overestimates. The residuals in $\rho$ are slightly offset, and this probably has a contribution from the small offset given by the $\Delta v$ scaling relation (recall that most of the pipelines rely on use of the scaling relation, which the BeSSP/GARSTEC reference here does not). The residuals for $M$ are also slightly off-center, though at a level much smaller than the uncertainty in the results.

The distribution of age-residuals is of course the most interesting. Normalizing the residuals by the uncertainties means that for the full cohort the errors in the results caused by the lack of metallicity data cannot be the cause of the skewness of the distribution (recall that here we plot the IRFM results). Plots of results from the smaller cohort with spectroscopic data show a similar-shaped distribution. The non-Gaussian nature of the distribution is mainly a result of the fact that, of all the properties, model dependences are most marked in the ages, i.e., differences in the physics of the grids result in different ages for the same inputs. These dependencies were explored in detail by Gai et al. (2011), and we see similar systematic effects here. Figure 11 helps to illustrate the grid-based systematics associated with the ages. It shows histograms of the normalized residuals in $t$-as per the normalized residuals in Figure 10-but for individual grids, and only those coupled to the same (YB) pipeline. Note that for clarity we have plotted each of the histograms by joining the midpoints of the bins. The offsets between histograms indicate that the systematics are no larger than the median formal uncertainties returned by the pipelines. 

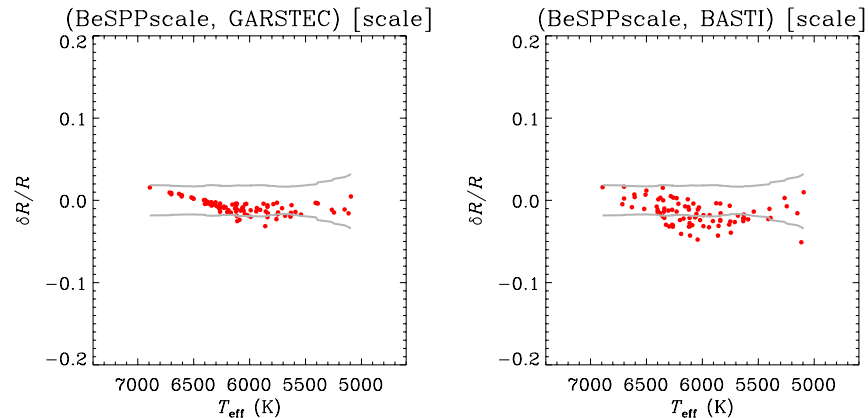

$T_{\text {eff }}(\mathrm{K})$
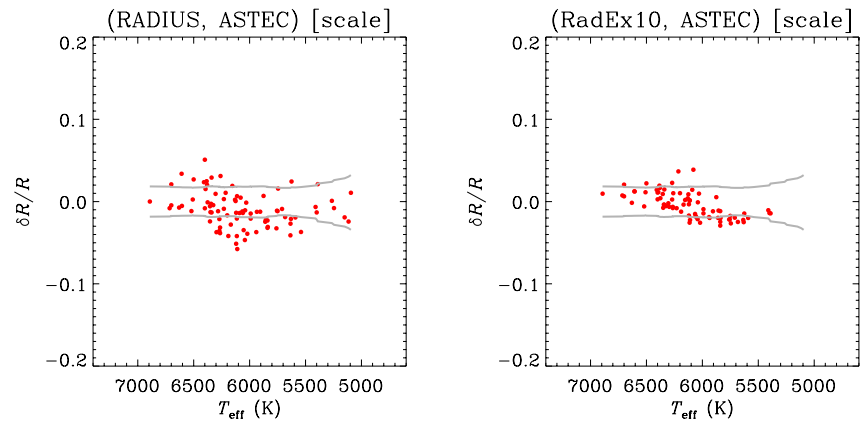

$T_{\text {eff }}(\mathrm{K})$

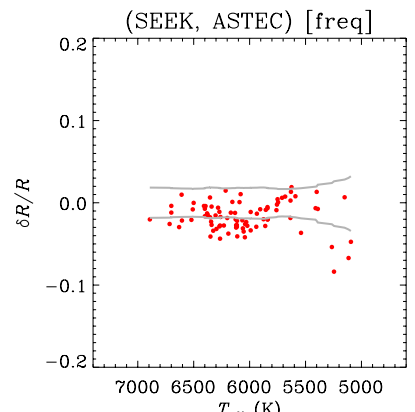

$T_{\text {eff }}(\mathrm{K})$

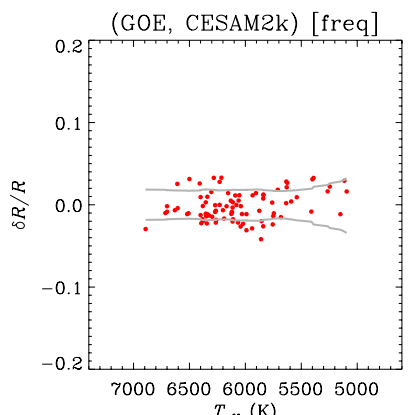

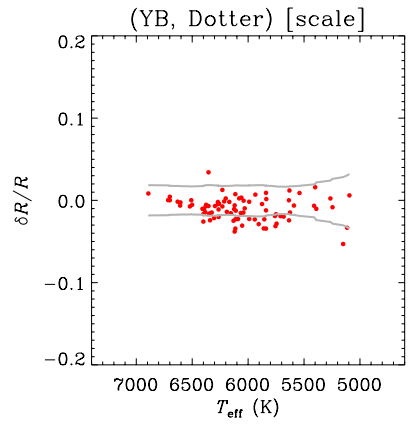
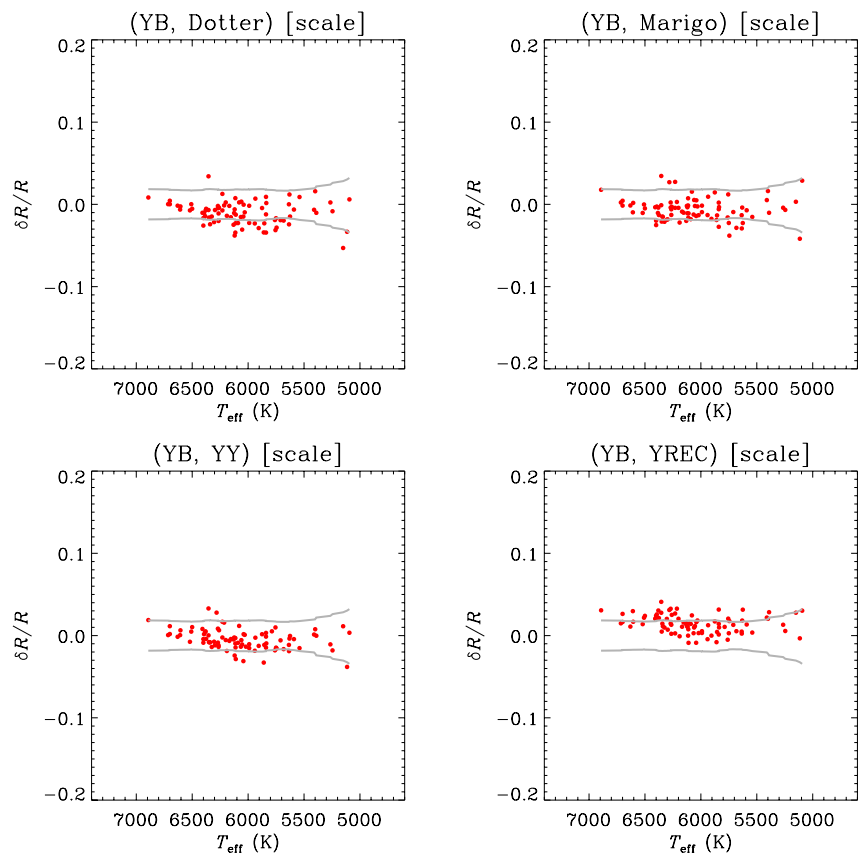

(YB, YREC2) [scale]
$T_{\text {eft }}(\mathrm{K})$

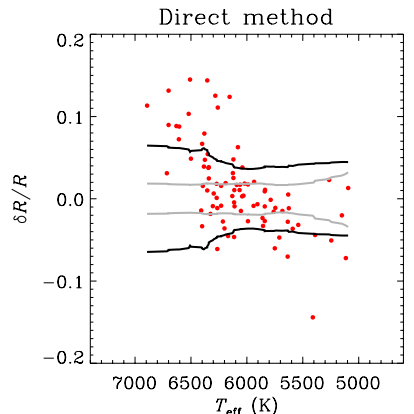

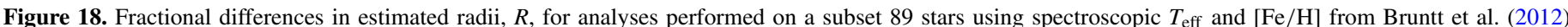

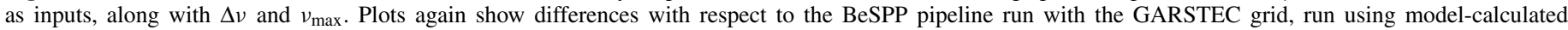

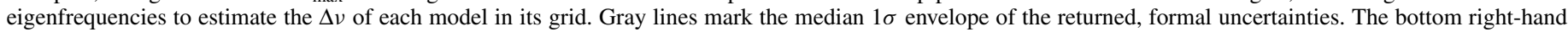
panel shows results from direct application of the scaling relations, the black lines showing the median $1 \sigma$ envelope on the resulting uncertainties.

(A color version of this figure is available in the online journal.)

The more pronounced negative tail in the age histogram in Figure 10 basically tells us that ages determined by GARSTEC are in general slightly higher than the average.

For the full cohort, the median standard deviations (scatter between grid-pipelines) - which provide a measure of the combined effect of the grid-based and pipeline-based errors - are approximately $4.5 \%$ in mass, $1.7 \%$ in radius, 0.006 dex in $\log g$, $1.3 \%$ in density, and $16 \%$ in age (similar for both the IRFM and SDSS results); for the smaller cohort with spectroscopic data they are around $3.7 \%, 1.3 \%, 0.005 \mathrm{dex}, 1.2 \%$, and $12 \%$, respectively.

The above measures of scatter are combined in quadrature with the individual formal uncertainties to yield the final uncertainties on the estimated properties. Owing to the much tighter constraints on $[\mathrm{Fe} / \mathrm{H}]$, the final uncertainties for the sample with spectroscopic data are, as expected, smaller than those on the full cohort. Median final uncertainties on the spectroscopic sample are $\approx 5.4 \%$ in mass, $2.2 \%$ in radius, 0.010 dex in $\log g, 2.8 \%$ in density, and $25 \%$ in age; results on common stars from the full cohort yield final median uncertainties of around $9.4 \%, 3.5 \%, 0.015 \mathrm{dex}, 3.3 \%$, and $32 \%$, respectively for the IRFM results, and slightly smaller values for the SDSS results (owing to the slightly smaller fractional
$T_{\text {eff }}$ uncertainties for the SDSS data). When all stars in the full cohort are taken, median final uncertainties for the IRFM results are $\approx 10.8 \%$ in mass, $4.4 \%$ in radius, 0.017 dex in $\log g, 4.3 \%$ in density, and $34 \%$ in age (again, slightly smaller for the SDSS results).

Whilst use of the seismic inputs $\Delta v$ and $v_{\max }$ has allowed much tighter constraints to be placed on the ages of most of these stars than would have been possible in the absence of such information, some of the uncertainties are large. Figure 12 plots histograms of the final (i.e., with the scatter contributions now included) uncertainties on the ages, and also the masses and radii, for the IRFM results. It will be possible to do much better for around 150 of these stars, by utilizing information from estimates of the individual frequencies.

In sum: the ages presented here are not the bottom line on what is possible using asteroseismology.

Nevertheless, ages have in most cases been estimated to a precision that is useful in a statistical or ensemble sense. Just over 70\% (SDSS results) and just under 60\% (IRFM results) of stars in the full cohort have final fractional age uncertainties that are $30 \%$ or better. This fraction increases to over $80 \%$ in the sample with spectroscopic data, which only reinforces the point that to fully utilize the diagnostic potential of the 

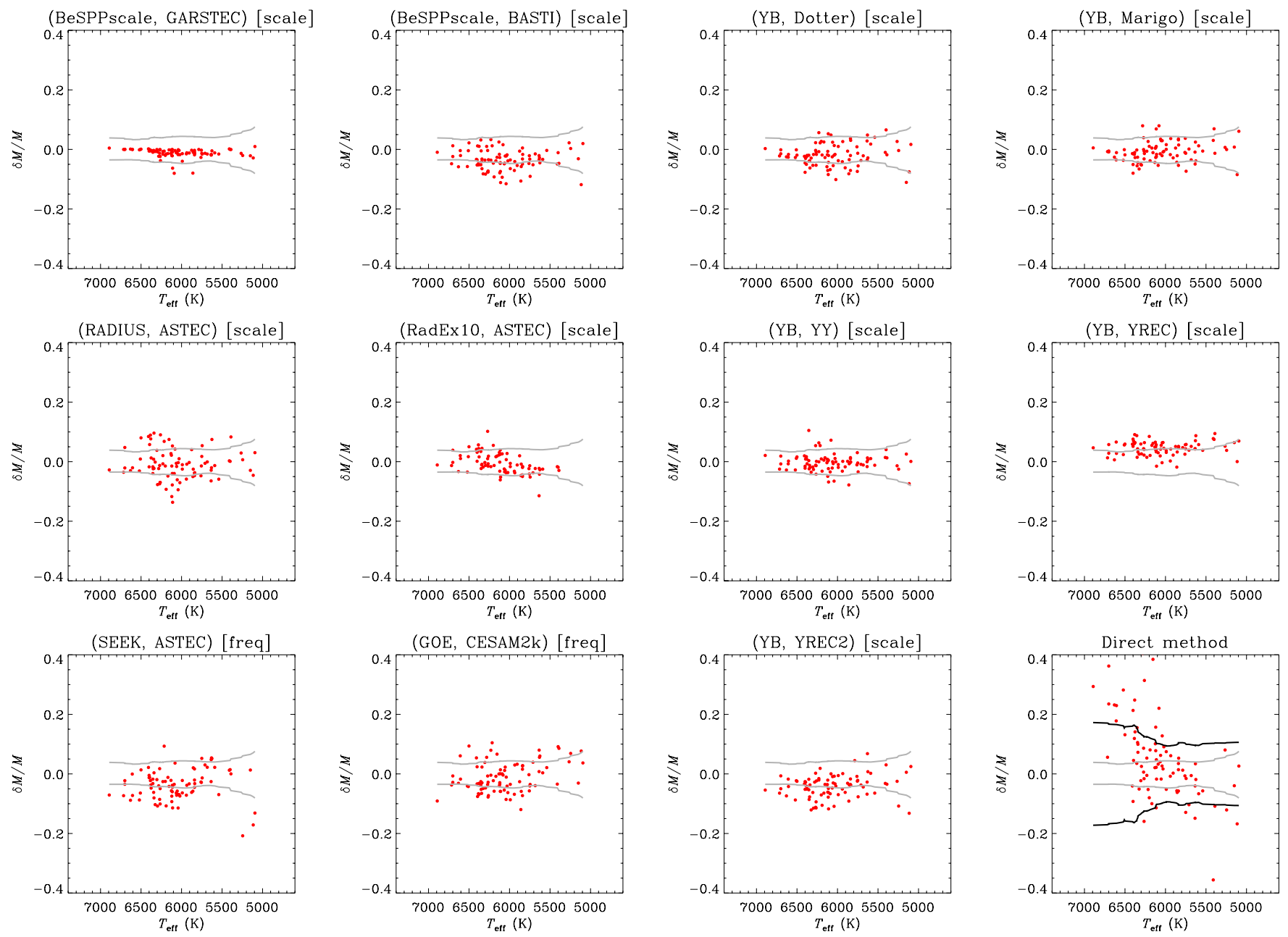

Figure 19. As per Figure 18, but for fractional differences in mass $M$.

(A color version of this figure is available in the online journal.)

seismic data (or any other data) for constraining the ages, good constraints on stellar composition are required. It should of course be borne in mind that the scatter found here will reflect model dependencies for the physics adopted in the grids we used, and that other choices (which could affect the ages) are possible. The final, quoted uncertainties on the ages presented here must be used/considered in any analysis: it is these values that capture the systematics due to the stellar model dependencies from the adopted physics in the commonly used grids we have used, and differences in the adopted pipeline methodologies. It should also be remembered that the ages come from one gridpipeline combination only, and age is the most model-dependent property.

Results from the Geneva Copenhagen Survey (GCS) provide a useful comparison of what is possible for field stars in the absence of results from seismology, when high-quality parallaxes, effective temperatures and metallicities are available (Nordström et al. 2004; Casagrande et al. 2011). An appropriate comparison is one made with results from the Bruntt et al. cohort with spectroscopic data, since metallicity information is available for all GCS targets. It should be borne in mind that ages and masses from the GCS are based on the use of two grids of models (Padova and BASTI), while in the present paper systematics stemming from different models come from the use of eleven grids. The GCS sample is also brighter than the KASC sample. Median age uncertainties for the Bruntt et al. cohort and the CGS are quite similar. As noted above, $80 \%$ of Bruntt et al. cohort have fractional ages uncertainties of $30 \%$ or better. However only about $50 \%$ of the stars in the GCS have errors $\leqslant 30 \%$.

\section{CONCLUSION}

We have presented a homogeneous asteroseismic analysis of over 500 solar-type main-sequence and sub-giant stars observed by Kepler. Stellar properties were estimated using two global asteroseismic parameters - the average large frequency separation, $\Delta v$, and the frequency of maximum oscillations power, $v_{\max }$ - and complementary photometric and spectroscopic data. Homogeneous sets of effective temperatures, $T_{\text {eff }}$, were available for the entire ensemble using available complementary photometry; spectroscopic estimates of $T_{\text {eff }}$ and $[\mathrm{Fe} / \mathrm{H}]$ were available from a homogeneous analysis of ground-based data on a subset totaling 87 stars.

We provide estimates of stellar radii, masses, surface gravities, mean densities and ages. We add a strong note of caution regarding the ages. The quoted age uncertainties are similar to those obtained in the best case scenario from isochrone fitting of field stars when parallaxes, metallicities and effective temperatures are known precisely (e.g., Nordström et al. 2004; Casagrande et al. 2011). Although the advantages and the potential of asteroseismology are obvious, many of the usual warnings 

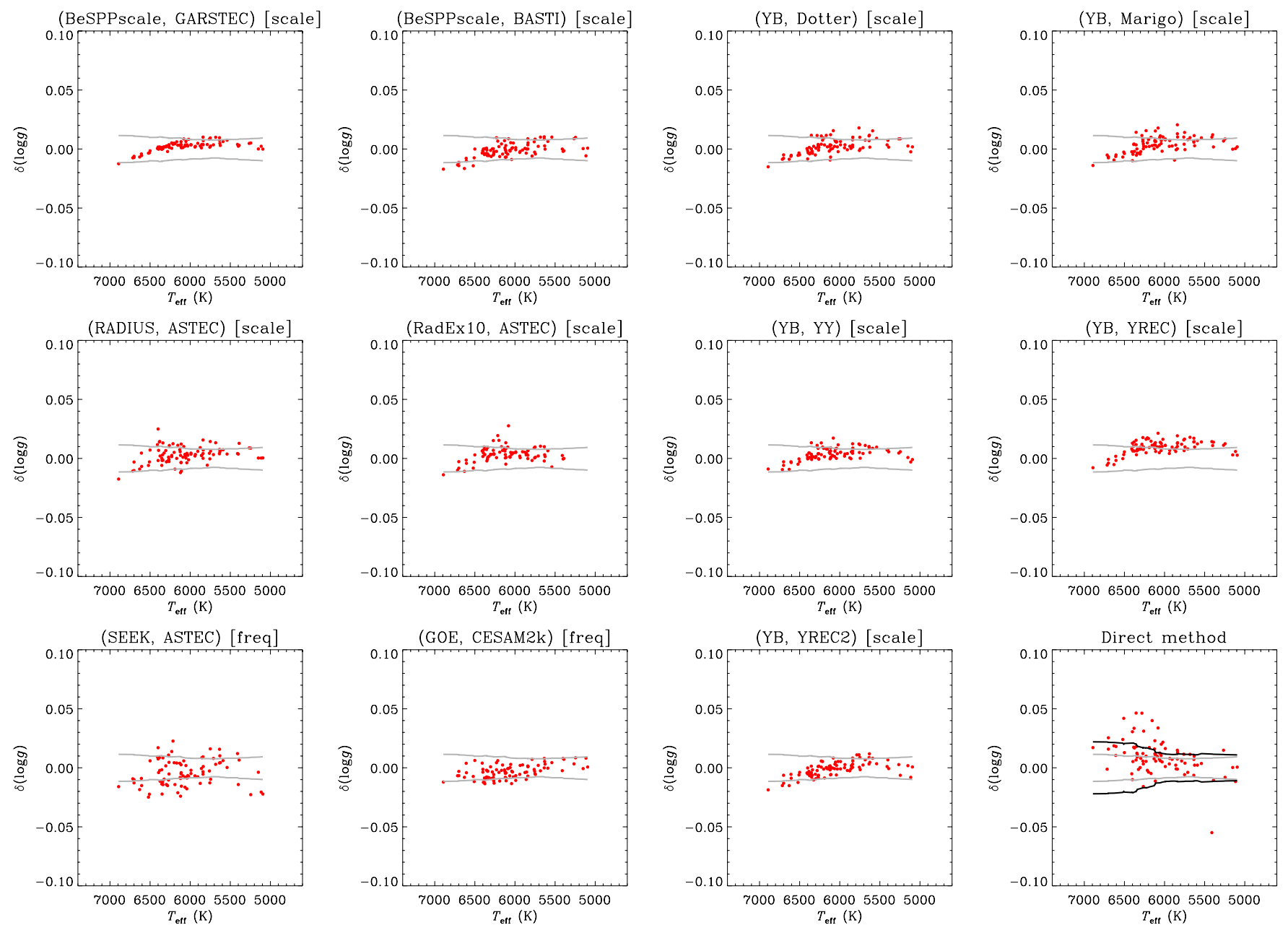

Figure 20. As per Figure 18, but for differences in $\log g$.

(A color version of this figure is available in the online journal.)

thus apply to the present sample. The ages in this paper will be useful for ensemble studies, but should be treated carefully when considered on a star-by-star basis. Because we used only two global asteroseismic parameters, future analyses using individual oscillation frequencies - which are important for asteroseismic estimation of ages - will offer significant improvements on up to 150 stars.

A future priority will be to collect further high-quality spectroscopy and photometry in appropriate colors (e.g., the Strömgren filters), in order to place tight constraints on the compositions of all stars in the sample. This should be possible from both episodic ground-based campaigns, and from utilizing data from planned spectroscopic surveys, e.g., APOGEE (Majewski et al. 2010). Gaia (see, e.g., Gilmore et al. 2012) will provide exquisite parallaxes on the cohort of stars in this paper. Since the asteroseismic data can provide accurate surface gravities, they can also play an important role in helping to calibrate spectroscopic analyses. A formal collaboration (APOKASC) has already been established between APOGEE and the KASC, with the full sample discussed in this paper having already been included in target planning.

We thank the anonymous referee for helpful comments that improved the paper. Funding for this Discovery mission is provided by NASA's Science mission Directorate. The authors wish to thank the entire Kepler team, without whom these results would not be possible. W.J.C. acknowledges the support of the UK Science and Technology Facilities Council (STFC). S.B. ackowledges support from NSF grant AST-1105930 and NASA grant NNX13AE70G. D.H. is supported by an appointment to the NASA Postdoctoral Program at Ames Research Center, administered by Oak Ridge Associated Universities through a contract with NASA. A.M.S. is partially supported by the International Reintegration Grant PIRG-GA-2009-247732, and the MICINN grant AYA2011-24704. L.G., W.H.B., and J.P.M. acknowledge support from Collaborative Research Center CRC 963 "Astrophysical Flow Instabilities and Turbulence" (Project A18), funded by the German Research Foundation (DFG). O.L.C. was an Henri Poincaré Fellow at the Observatoire de la Côte d'Azur. The Henri Poincaré Fellowship is funded by the Conseil Général des Alpes-Maritimes and the Observatoire de la Côte d'Azur. J.M.-Ż. acknowledges support from MNiSW grant N N203 405139. T.S.M. acknowledges NASA grant NNX13AE91G. R.A.G. acknowledges the funding from the European Community's Seventh Framework Programme (FP7/2007-2013) under grant agreement no. 269194 (IRSES/ASK). R.A.G. and B.M. acknowledge support from the ANR program IDEE "Interaction Des Etoiles et des Exoplanètes" (Agence Nationale de la Recherche, France). S.H. acknowledges financial support from the Netherlands Organisation for Scientific Research (NWO). 

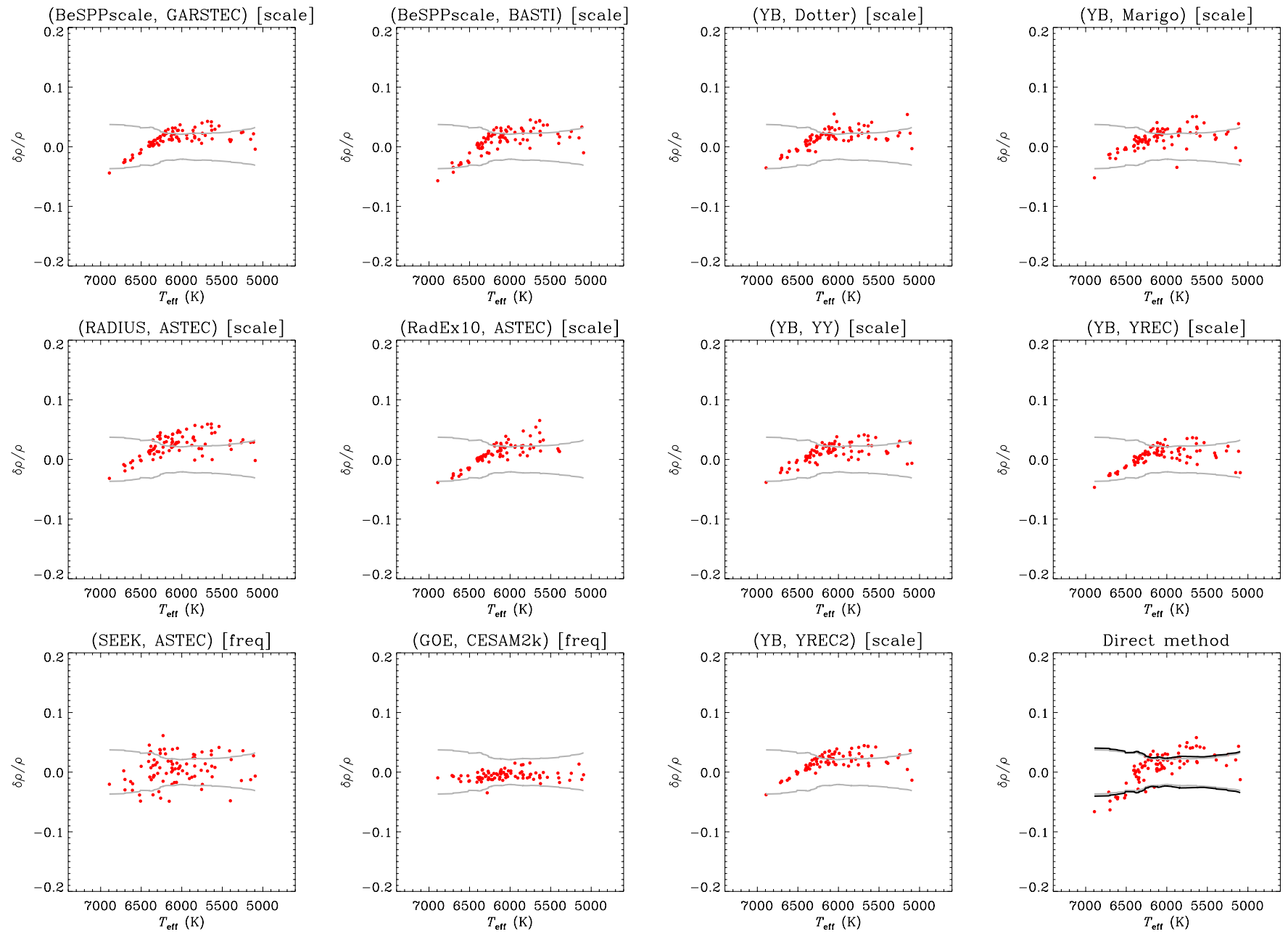

Figure 21. As per Figure 18, but for fractional differences in average density $\rho$.

(A color version of this figure is available in the online journal.)

Funding for the Stellar Astrophysics Centre is provided by The Danish National Research Foundation (grant agreement No.: DNRF106). The research is supported by the ASTERISK project (ASTERoseismic Investigations with SONG and Kepler) funded by the European Research Council (grant agreement No.: 267864). The research leading to these results has received funding from the European Community's Seventh Framework Programme (FP7/2007-2013) under grant agreement no. 312844 (SPACEINN).

We are also grateful for support from the International Space Science Institute (ISSI).

\section{APPENDIX \\ COMPARISON PLOTS FOR DIFFERENT GRID-PIPELINE COMBINATIONS}

Figures 13 through 17 show comparisons of results from the different grid-pipeline combinations, for analyses performed on the entire ensemble, with $\Delta v, v_{\max }$, the photometric (IRFM) $T_{\text {eff }}$, and field $[\mathrm{Fe} / \mathrm{H}]$ values used as inputs. The plots show differences with respect to a common reference set of results, those given by BeSPP using the GARSTEC grid and modelcalculated eigenfrequencies to estimate the $\Delta v$ of each model (the "frequency" mode of this pipeline).
The plotted differences are fractional differences in $R, M, \rho$ and age $t$; and absolute differences in $\log g$. The gray lines mark envelopes corresponding to the median of the $1 \sigma$ uncertainties returned by all grid pipelines, with medians calculated in 10-target batches sorted on the independent variable used for the plots ( $T_{\text {eff }}$ for $R, M, \log g$ and $\rho$; and $\Delta v$ for $\left.t\right)$. The results shown in the lower right-hand plots are those given by direct application of the scaling relations (note there is no directmethod estimate of age $t$ ). The thick black lines mark the $1 \sigma$ uncertainty envelopes for the direct method. We note that SEEK, RadEx 10 and GOE were coupled to grids whose sampling was less dense for more evolved (generally lower $T_{\text {eff }}$ ) stars: hence, for those cases some results have not been returned on targets in this part of the parameter space.

The direct-method results are typically more scattered, but on the whole consistent with the larger uncertainties expected from basic error-propagation. The uncertainties are smaller for the grid-based searches because the solutions are constrained to satisfy stellar evolution theory, hence only a narrow range of outcomes is permitted. The native fractional uncertainties on $v_{\max }$ are, on average, about twice the size of those on $\Delta v$. This acts to drive up the direct-method uncertainties, most notably on $R$ and $M$. The direct-method uncertainties for $\rho$, which are determined solely by uncertainties in $\Delta v$, are in contrast seen to match quite closely the uncertainties given by the grid-based searches. 

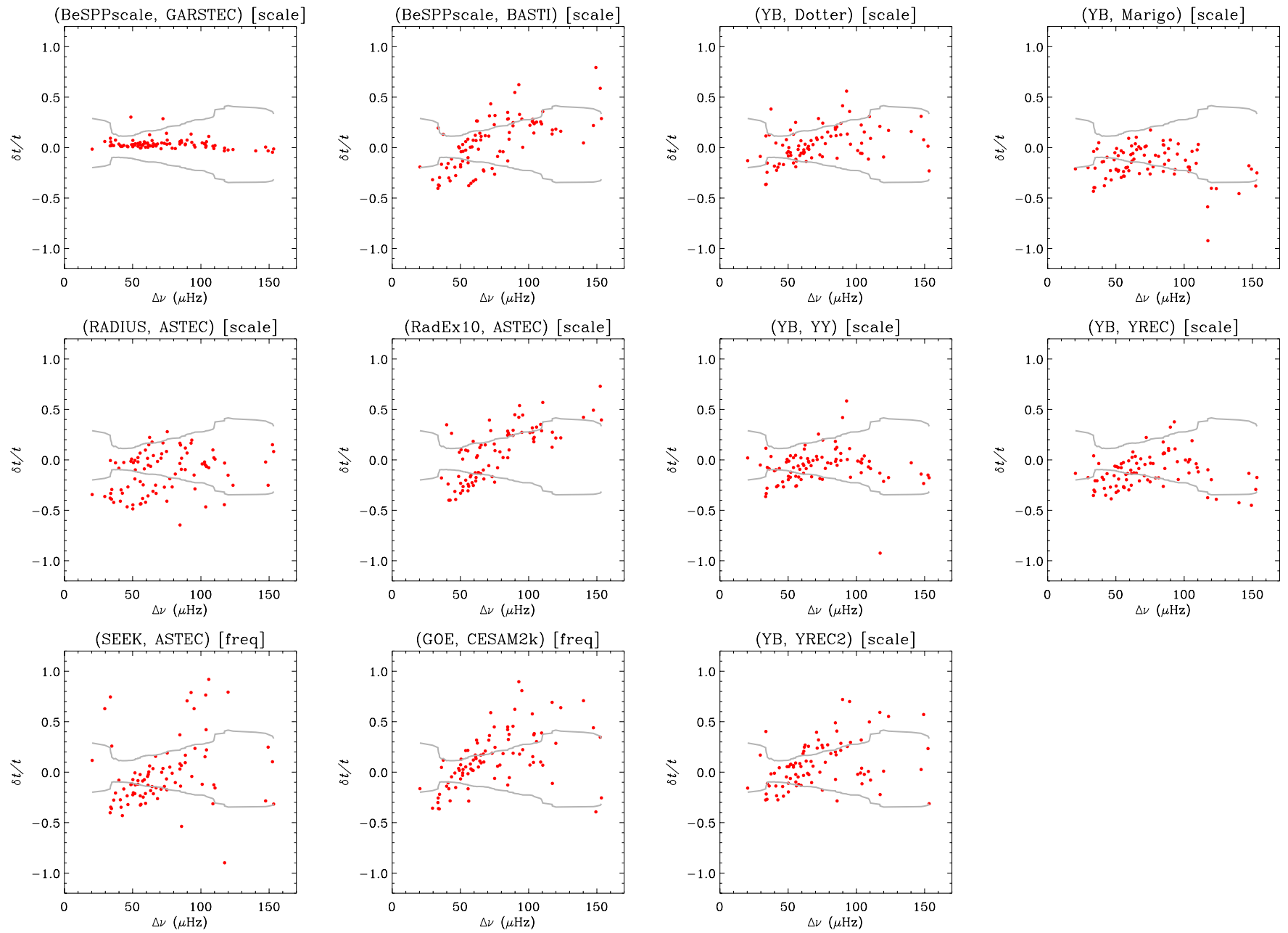

Figure 22. As per Figure 18, but for fractional differences in age, $t$, and with $\Delta v$ as the independent variable for the plot.

(A color version of this figure is available in the online journal.)

Figures 18 through 22 show similar visual comparisons of results, this time for analyses performed on the cohort that had spectroscopic $T_{\text {eff }}$ and $[\mathrm{Fe} / \mathrm{H}]$ available.

Inspection of the results shows that in most cases the plotted differences lie within the typical, median $1 \sigma$ uncertainty envelope. We see clearly the impact of adopting the scaling relations to compute $\Delta v$, i.e., as manifested in the boomerang-shaped trends which are most notable in the density plots, but also present in radius, gravity and mass. These trends are noticeably lacking when pipelines that used calculated eigenfrequencies are compared (e.g., see the flat density difference for the GOE pipeline, in Figure 16. We note that, at the level of precision of these data, the boomerang-shaped differences lie largely within the median $1 \sigma$ envelopes.

There is also evidence of model dependence in the results. For example, masses and radii returned by GOE are both offset positively, by different fractional amounts, relative to the reference BeSPP results. These offsets combine to give a small negative, albeit flat (see above) offset in density. Also noteworthy is the larger internal scatter shown by the SEEK results (all properties), relative to the internal scatter shown by the other pipelines. SEEK is coupled to a grid with multiple values of the mixing length parameter, $\alpha$. However, not all subgrids constructed with a given $\alpha$ cover the full range of metallicity. The increased scatter in the SEEK estimates is a result of this non-uniform sampling of the $\alpha-[\mathrm{Fe} / \mathrm{H}]$ space. It is also worth remarking again on the scatter shown by the age estimates.

\section{REFERENCES}

Adelberger, E. G., Austin, S. M., Bahcall, J. N., et al. 1998, RvMP, 70, 1265 Adelberger, E. G., García, A., Robertson, R. G. H., et al. 2011, RvMP, 83, 195 Alexander, D. R., \& Ferguson, J. W. 1994, ApJ, 437, 879

Appourchaux, T., Michel, E., Auvergne, M., et al. 2008, A\&A, 488, 705

Asplund, M., Grevesse, N., \& Sauval, A. J. 2005, in ASP Conf. Ser. 336, Cosmic Abundances as Records of Stellar Evolution and Nucleosynthesis, ed. T. G. Barnes III \& F. N. Bash (San Francisco, CA: ASP), 25

Asplund, M., Grevesse, N., Sauval, A. J., \& Scott, P. 2009, ARA\&A, 47, 481

Bahcall, J. N. 1989, Neutrino Astrophysics (Cambridge: Cambridge Univ. Press) Bahcall, J. N., \& Pinsonneault, M. H. 1992, RvMP, 64, 885

Basu, S., Chaplin, W. J., \& Elsworth, Y. 2010, ApJ, 710, 1596

Basu, S., Verner, G. A., Chaplin, W. J., \& Elsworth, Y. 2012, ApJ, 746, 76

Batalha, N. M., Borucki, W. J., Bryson, S. T., et al. 2011, ApJ, 729, 27

Bazot, M., Bourguignon, S., \& Christensen-Dalsgaard, J. 2012, MNRAS, 427, 1847

Bedding, T. R. 2011, in Asteroseismology, Vol. 23, Canary Islands Winter School of Astrophysics, ed. P. Pallé (Cambridge: Cambridge Univ. Press), in press (arXiv:1107.1723)

Belkacem, K., Goupil, M. J., Dupret, M. A., et al. 2011, A\&A, 530, 142

Bonanno, A., Benatti, S., Claudi, R., et al. 2008, ApJ, 676, 1248

Brogaard, K., VandenBerg, D. A., Bruntt, H., et al. 2012, A\&A, 543, A106

Brown, T. M., Gilliland, R. L., Noyes, R. W., \& Ramsey, L. W. 1991, ApJ, 368, 599

Brown, T. M., Latham, D. W., Everett, M. E., \& Esquerdo, G. A. 2011, AJ, 142,111 
Bruntt, H., Basu, S., Smalley, B., et al. 2012, MNRAS, 423, 122

Campante, T. L., Karoff, C., Chaplin, W. J., et al. 2010, MNRAS, 408, 542

Carter, J. A., Agol, E., Chaplin, W. J., et al. 2012, Sci, 337, 556

Casagrande, L., Ramírez, I., Meléndez, J., et al. 2010, A\&A, 512, 54

Casagrande, L., Schönrich, R., Asplund, M., et al. 2011, A\&A, 530, 138

Cassisi, S., Salaris, M., \& Irwin, A. W. 2003, ApJ, 588, 862

Caughlan, G. R., \& Fowler, W. A. 1988, ADNDT, 40, 283

Chaplin, W. J., Houdek, G., Appourchaux, T., et al. 2008, A\&A, 485, 813

Chaplin, W. J., Kjeldsen, H., \& Christensen-Dalsgaard, J. 2011, Sci, 332, 213

Chaplin, W. J., \& Miglio, A. 2013, ARA\&A, 51, 353

Chaplin, W. J., Sanchis-Ojeda, R., Campante, T. L., et al. 2013, ApJ, 766, 101

Christensen-Dalsgaard, J. 2008, Ap\&SS, 316, 13

Christensen-Dalsgaard, J. 1993, in ASP Conf. Ser. 42, Proc. GONG 1992: Seismic Investigation of the Sun and Stars, ed. T. M. Brown (San Francisco, CA: ASP), 347

Christensen-Dalsgaard, J., \& Gough, D. O. 1984, in Solar Seismology from Space, ed. R. K. Ulrich, J. Harvey, E. J. Rhodes, Jr., \& J. Toomre (JPL Publ. 84-84; Pasadena, CA: JPL), 199

Christensen-Dalsgaard, J., \& Thompson, M. J. 1997, MNRAS, 284, 527

Creevey, O. L., Doğan, G., Frasca, A., et al. 2012, A\&A, 537, 111

Creevey, O. L., Theévenin, F., Basu, S., et al. 2013, MNRAS, 431, 2419

Cunha, M. S., Aerts, C., Christensen-Dalsgaard, J., et al. 2007, A\&ARv, 14,217

Demarque, P., Guenther, D. B., Li, L. H., Mazumdar, A., \& Straka, C. W. 2008, Ap\&SS, 316, 31

Demarque, P., Woo, J.-H., Kim, Y.-C., \& Yi, S. K. 2004, ApJS, 155, 667

Doğan, G., Metcalfe, T. S., Deheuvels, S., et al. 2013, ApJ, 763, 49

Dotter, A., Chaboyer, B., Jevremović, D., et al. 2008, ApJS, 178, 89

Drimmel, R., Cabrera-Lavers, A., \& López-Corredoira, M. 2003, A\&A, 409, 205

Dziembowski, W. A., Paterno, L., \& Ventura, R. 1988, A\&A, 200, 213

Eggleton, P. P., Faulkner, J., \& Flannery, B. P. 1973, A\&A, 23, 325

Ferguson, J. W., Alexander, D. R., Allard, F., et al. 2005, ApJ, 623, 585

Gai, N., Basu, S., Chaplin, W. J., \& Elsworth, Y. 2011, ApJ, 730, 63

García, R. A., Hekker, S., Stello, D., et al. 2011, MNRAS, 414, 6

Gilliland, R. L., Brown, T. M., Christensen-Dalsgaard, J., et al. 2010a, PASP, 122,131

Gilliland, R. L., Jenkins, J. M., Borucki, W. J., et al. 2010b, ApJL, 713, L160

Gilliland, R. L., Marcy, G. W., Rowe, J. F., et al. 2013, ApJ, 766, 40

Gilmore, G., Randich, S., Asplund, M., et al. 2012, Msngr, 147, 25

Girardi, L., Bressan, A., Bertelli, G., \& Chiosi, C. 2000, A\&AS, 141, 371

Grevesse, N., \& Noels, A. 1993, PhST, 47, 133

Grevesse, N., \& Sauval, A. J. 1998, SSRv, 85, 161

Gruberbauer, M., Guenther, D. B., \& Kallinger, T. 2012, ApJ, 749, 109

Gruberbauer, M., Guenther, D. B., McLeod, K., \& Kallinger, T. 2013, MNRAS, 435,242

Hekker, S., Broomhall, A.-M., Chaplin, W. J., et al. 2010, MNRAS, 402, 2049

Hekker, S., Elsworth, Y., Basu, S., et al. 2013, MNRAS, 434, 1668

Howell, S. B., Rowe, J. F., Bryson, S. T., et al. 2012, ApJ, 746, 123

Huber, D., Bedding, T. R., Stello, D., et al. 2011, ApJ, 743, 143
Huber, D., Ireland, M. J., Bedding, T. R., et al. 2012, ApJ, 760, 32

Huber, D., Stello, D., Bedding, T. R., et al. 2009, CoAst, 160, 74

Jenkins, J. M., Caldwell, D. A., Chandraekaren, H., et al. 2010, ApJL, 713, L87

Kjeldsen, H., \& Bedding, T. R. 1995, A\&A, 293, 87

Kjeldsen, H., Bedding, T. R., \& Christensen-Dalsgaard, J. 2008, ApJL, 683, L175

Kurucz, R. L. 1991, in Proc. Advanced Research Workshop on Stellar Atmospheres: Beyond Classical Models, Trieste, Italy, ed. L. Crivellari, I. Hubený, \& D. G. Hummer (Dordrecht: Reidel), 441

Landré, V., Prantzos, N., Aguer, P., et al. 1990, A\&A, 240, 85

Majewski, S. R., Wilson, J. C., Hearty, F., Schiavon, R. R., \& Skrutskie, M. F. 2010, in IAU Symp. 265, Chemical Abundances in the Universe: Connecting First Stars to Planets, ed. K. Cunha, M. Spite, \& B. Barbuy (Cambridge: Cambridge Univ. Press), 480

Marigo, P., Girardi, L., Bressan, A., et al. 2008, A\&A, 482, 883

Marques, J. P., Goupil, M. J., Lebreton, Y., et al. 2013, A\&A, 549, A74

Mathur, S., García, R. A., Régulo, C., et al. 2010, A\&A, 511, 46

Mathur, S., Metcalfe, T. S., Woitaszek, M., et al. 2012, ApJ, 749, 152

Metcalfe, T. S., Chaplin, W. J., Appourchaux, T., et al. 2012, ApJL, 748, L10

Metcalfe, T. S., Monteiro, M. J. P. F. G., Thompson, M. J., et al. 2010, ApJ, 723, 1583

Michel, E., \& Baglin, A. 2012, in Second CoRoT Symposium: Transiting planets, Vibrating Stars and Their Connection, ed. A. Baglin, M. Deleuil, E. Michel, \& C. Moutou, in press (arXiv:1202.1422)

Michel, E., Baglin, A., Auvergne, M., et al. 2008, Sci, 322, 558

Miglio, A. 2012, in Red Giants as Probes of the Structure and Evolution of the Milky Way, ed. A. Miglio, J. Montalbàn, \& A. Noels (Berlin: Springer), 11

Miglio, A., Brogaard, K., Stello, D., et al. 2012, MNRAS, 419, 2077

Mihalas, D., Däppen, W., \& Hummer, D. G. 1988, ApJ, 331, 815

Morel, O., \& Lebreton, Y. 2008, ApS\&S, 316, 61

Mosser, B., \& Appourchaux, T. 2009, A\&A, 508, 877

Mosser, B., Michel, E., Belkacem, K., et al. 2013, A\&A, 550, 126

Nordström, B., Mayor, M., Andersen, J., et al. 2004, A\&A, 418, 989

Pietrinferni, A., Cassisi, S., Salaris, M., \& Castelli, F. 2004, ApJ, 612, 168

Pinsonneault, M. H., An, D., Molenda-Żakowicz, J., et al. 2012, ApJS, 199, 30

Quirion, P.-O., Christensen-Dalasgaard, J., \& Arentoft, T. 2010, ApJ, 725,2176

Roxburgh, I. W. 2009, A\&A, 506, 435

Silva Aguirre, V., Basu, S., Brandão, I. M., et al. 2013, ApJ, 769, 141

Silva Aguirre, V., Casagrande, L., Basu, S., et al. 2012, ApJ, 757, 99

Silva Aguirre, V., Chaplin, W. J., Ballot, J., et al. 2011, ApJL, 740, L2

Skrutskie, M. F., Cutri, R. M., Stiening, R., et al. 2006, AJ, 131, 1163

Stello, D., Bruntt, H., Preston, H., \& Buzasi, D. 2008, ApJL, 674, L53

Stello, D., Chaplin, W. J., Bruntt, H., et al. 2009, ApJ, 700, 1589

Straniero, O. 1988, A\&AS, 76, 157

Tassoul, M. 1980, ApJS, 43, 469

Torres, G., Fischer, D. A., Sozzetti, A., et al. 2012, ApJ, 757, 161

Ulrich, R. K. 1986, ApJL, 306, L37

Verner, G. A., Elsworth, Y., \& Chaplin, W. J. 2011, MNRAS, 415, 3539

Weiss, A., \& Schlattl, H. 2008, ApS\&S, 316, 99

White, T. R., Bedding, T. R., Stello, D., et al. 2011, ApJ, 743, 161 